\title{
Rational quadrature formulas on the unit circle with prescribed nodes and maximal domain of validity
}
A. Bultheel, $1,5,6$
P. González-Vera ${ }^{2,5}$
E. Hendriksen ${ }^{3}$
O. $\mathrm{Njåstad}^{4}$

\begin{abstract}
This paper is concerned with rational Szegö quadrature formulas to approximate integrals of the form $I_{\mu}(f)=\int_{-\pi}^{\pi} f\left(e^{i \theta}\right) d \mu(\theta)$ by a formula like $I_{n}(f)=\sum_{k=1}^{n} \lambda_{k} f\left(z_{k}\right)$ where the weights $\lambda_{k}$ are positive and the nodes $z_{k}$ are carefully chosen on the complex unit circle. It will be shown that for a given set of poles, the quadrature formulas can be chosen to be exact in certain subspaces of rational functions of dimension $2 n$. Also the problem where one node (Radau) or two nodes (Lobatto) are prefixed will be analyzed and the corresponding rational rational Szegö-Radau and rational Szegö-Lobatto quadrature formulas shall be characterized.
\end{abstract}

Key words: rational Szegö-Radau quadrature, rational Szegö-Lobatto quadrature, maximal domain of validity,

2000 MSC: 42C05 41A55

$\overline{1}$ Department of Computer Science, K.U.Leuven, Belgium.

2 Department of Mathematical Analysis, La Laguna University, Tenerife, Spain.

3 Beerstratenlaan 23, 2421GN Nieuwkoop, The Netherlands.

4 Department of Math. Sc., Norwegian Univ. of Science and Technology, Trondheim, Norway.

5 This work is partially supported by the Direción General de Investigación, Ministerio de Educación y Ciencia, under grant MTM2005-08571.

6 The work of this author is partially supported by the Fund for Scientific Research (FWO), projects "RAM: Rational modelling: optimal conditioning and stable algorithms", grant \#G.0423.05 and the Belgian Network DYSCO (Dynamical Systems, Control, and Optimization), funded by the Interuniversity Attraction Poles Programme, initiated by the Belgian State, Science Policy Office. The scientific responsibility rests with the author. 


\section{Introduction}

It is well known that $n$-point Gauss quadrature formulas to approximate an integral $\int f(x) d \mu(x)$ with positive measure $\mu$ are quadrature rules with positive weights that integrate exactly all polynomials up to degree $2 n-1$, a subspace of dimension $2 n$. This is obtained by choosing the $n$ weights and the $n$ nodes in such a way that all polynomials of the maximal possible degree are integrated exactly. The set of polynomials of degree at most $2 n-1$ is a maximal domain of validity for these quadrature formulas. The nodes should be chosen to be zeros of a polynomial orthogonal with respect to the measure $\mu$.

Szegó quadrature formulas were used in [8] (see also [9]) and further discussed in Jones et al. in [12]. These are quadrature formulas of a similar nature approximating integrals where $\mu$ is supported on the complex unit circle. In this case a maximal domain of validity is the set of Laurent polynomials spanned by $\left\{z^{k}: k=-(n-1), \ldots, n-1\right\}$, a space of dimension $2 n-1$. The $n$ positive weights and the $n$ nodes on the unit circle can not be taken as the zeros of orthogonal Szegő polynomials (with respect to the inner product induced by the measure $\mu$ ) because they have all their zeros inside the open unit disk. The nodes are zeros of so called paraorthogonal polynomials. Since there are $2 n$ parameters and a maximal domain of validity of dimension $2 n-1$, one may wonder if there is not a subspace of the space of Laurent polynomials of dimension $2 n$ for which the $n$-point Szegö quadrature formulas are exact, and indeed there is, as was recently shown in [14].

Szegö-Radau and Szegö-Lobatto formulas is a term coined by Jagels and Reichel in [11] and they refer to Szegö quadrature formulas in which one or two points respectively on the unit circle are fixed to be nodes of the quadrature formula.

Rational Szegő quadrature formulas were introduced in [4] by the authors and generalize the Szegö quadrature formulas in the sense that not only subspaces of Laurent polynomials are integrated exactly but more general subspaces of rational functions with prescribed poles.

In this paper, we engage into the study of rational Szegö-Radau and rational Szegö-Lobatto quadrature formulas, thus generalizing to some extend the results of [11] to the rational case.

The paper is arranged as follows. In Section 2 we recall the necessary results from the literature. In the subsequent Section 3 we show that rational Szegö quadrature formulas integrate exactly all rational functions from certain subspaces of dimension $2 n$. In Section 4 we characterize the rational Szegő quadrature formulas for which one node on the unit circle has been fixed and in Section 6 we analyze the Lobatto case when two points are fixed. In both cases quadrature formulas with positive weights will be derived. In an intermediate Section 5 a link between the 
rational para-orthogonal functions and the reproducing kernels for the subspaces of rational functions will be given.

We shall use the following notation for the unit circle, its interior and its exterior

$$
\mathbb{T}=\{z \in \mathbb{C}:|z|=1\}, \quad \mathbb{D}=\{z \in \mathbb{C}:|z|<1\}, \quad \mathbb{E}=\{z \in \mathbb{C}:|z|>1\}
$$

For an integral of the form $\int_{-\pi}^{\pi} F(z) d \mu(\theta)$, it is always assumed that $z=e^{i \theta}$.

Throughout the paper, $\mu$ will denote a positive Borel measure which defines the inner product

$$
\langle f, g\rangle_{\mu}=\int_{-\pi}^{\pi} f(z) \overline{g(z)} d \mu(\theta)=\int_{-\pi}^{\pi} f(z) g_{*}(z) d \mu(\theta),
$$

where for any function $f$, the substar conjugate represents a reflection in the unit circle: $f_{*}(z)=$ $\overline{f(1 / \bar{z})}$. Note that $f_{*}(z)=\overline{f(z)}$ if $z \in \mathbb{T}$.

By $\Pi_{n}$ we denote the set of polynomials whose degree is at most $n$.

$I_{\mu}(f)$ will refer to the integral $\int_{-\pi}^{\pi} f\left(e^{i \theta}\right) d \mu(\theta)$ which we also write as $\int_{-\pi}^{\pi} f(z) d \mu(\theta)$ where it is understood that $z=e^{i \theta}$. A quadrature formula with $n$ nodes will be denoted by $I_{n}(f)$ and is assumed to be of the form $\sum_{k=1}^{n} \lambda_{k} f\left(z_{k}\right)$ with weights $\lambda_{1}, \ldots, \lambda_{n}$ and nodes $z_{1}, \ldots, z_{n}$ in $\mathbb{T}$.

\section{Preliminary results}

Let $\alpha=\left\{\alpha_{k}\right\}_{1}^{\infty}$ be a given sequence in the interior of the unit disk $\mathbb{D}$.

For each of these $\alpha_{i} \in \mathbb{D}$, define the Blaschke factor

$$
\zeta_{i}(z)=\eta_{i} \frac{\alpha_{i}-z}{1-\bar{\alpha}_{i} z}, \quad \eta_{i}=\frac{\bar{\alpha}_{i}}{\left|\alpha_{i}\right|} \text { if } \alpha_{i} \neq 0 \text { and } \eta_{i}=-1 \text { if } \alpha_{i}=0
$$

We also need the Blaschke products

$$
B_{0}=1, \quad B_{n}=B_{n-1} \zeta_{n}, \quad n \geq 1
$$


Let $\mathcal{L}_{n}=\operatorname{span}\left\{B_{k}: k=0,1, \ldots, n\right\}$ so that with

$$
\omega_{0}=\pi_{0}=1 \text { and } \omega_{k}(z)=\prod_{j=1}^{k}\left(z-\alpha_{j}\right) \text { and } \pi_{k}(z)=\prod_{j=1}^{k}\left(1-\bar{\alpha}_{j} z\right), k=1,2, \ldots,
$$

it can be characterized as

$$
\mathcal{L}_{n}=\left\{f=\frac{P}{\pi_{n}}: P \in \Pi_{n}\right\}
$$

Note that we can write for $n \geq 1$

$$
B_{n}(z)=\gamma_{n} \frac{\omega_{n}(z)}{\pi_{n}(z)}, \quad \gamma_{n}=(-1)^{n} \prod_{j=1}^{n} \eta_{j}
$$

From the substar conjugate $f_{*}(z)=\overline{f(1 / \bar{z})}$ we can define

$$
\mathcal{L}_{n *}=\left\{f: f_{*} \in \mathcal{L}_{n}\right\} \quad \text { so that } \quad \mathcal{L}_{n *}=\left\{f: f=\frac{Q}{\omega_{n}}: Q \in \Pi_{n}\right\} .
$$

Furthermore for $p$ and $q$ nonnegative integers we set

$$
\mathcal{R}_{p, q}=\mathcal{L}_{p *}+\mathcal{L}_{q}=\left\{f=\frac{P}{\omega_{p} \pi_{q}}: P \in \Pi_{p+q}\right\}=\operatorname{span}\left\{B_{k}: k=-p, \ldots,-1,0,1, \ldots, q\right\},
$$

where we introduced the notation $B_{-k}(z)=B_{k *}(z)=1 / B_{k}(z)$.

Note that $\mathcal{R}=\bigcup_{p, q \geq 0} \mathcal{R}_{p, q}$ is dense in the class of continuous functions with respect to the $L_{r}$ norm, $1 \leq r \leq \infty$, if and only if $\sum_{n}\left(1-\left|\alpha_{n}\right|\right)=\infty$. See [1] (with an adaptation for coinciding $\alpha$ 's).

Finally we introduce the notation $\mathcal{L}_{n}(\alpha)$ to denote the set of functions in $\mathcal{L}_{n}$ that vanish in $\alpha$ : $\mathcal{L}_{n}(\alpha)=\left\{f \in \mathcal{L}_{n}: f(\alpha)=0\right\}$.

In this paper we shall be concerned with the estimation of the integral

$$
I_{\mu}(f)=\int_{-\pi}^{\pi} f\left(e^{i \theta}\right) d \mu(\theta)
$$


by means of an $n$-point quadrature rule

$$
I_{n}(f)=\sum_{j=1}^{n} \lambda_{j} f\left(z_{j}\right), \quad z_{j} \neq z_{k}, \text { if } j \neq k, \text { and } z_{j} \in \mathbb{T}, j=1, \ldots, n \text {. }
$$

The nodes $\left\{z_{j}\right\}_{j=1}^{n}$ and weights $\left\{\lambda_{j}\right\}_{j=1}^{n}$ in (2.1) are to be determined such that

$$
I_{\mu}(f)=I_{n}(f), \quad \forall f \in \mathcal{R}_{p(n), q(n)}
$$

with $q(n)+p(n)$ as large as possible. The basic requirement we are assuming for $\mu$ is that the integrals (rational moments)

$$
\mu_{k}=\int_{-\pi}^{\pi} \overline{B_{k}(z)} d \mu(\theta)
$$

must exist for any integer $k$. Observe that $B_{-k}(z)=B_{k *}(z)=\overline{B_{k}(z)}$ for $z \in \mathbb{T}$, so that $\mu_{-k}=\bar{\mu}_{k}$.

Orthogonal rational functions on the unit circle (rational Szegő functions) play a fundamental role in the construction of $I_{n}(f)$.

Indeed, for $n \geq 1$, let $\left\{\phi_{j}\right\}_{0}^{n}$ with $\phi_{j} \in \mathcal{L}_{j} \backslash \mathcal{L}_{j-1}$ be an orthonormal basis for $\mathcal{L}_{n}$, obtained by applying the Gram-Schmidt process to the basis $B_{0}, B_{1}, \ldots, B_{n}$. Let $\phi_{n}(z)=\sum_{k=0}^{n} a_{k} B_{k}(z)$, then $a_{n}$ is called the leading coefficient (with respect to the basis $\left\{B_{k}\right\}$ ). The $\phi_{j}$ are only defined up to a constant of modulus 1 . To make it unique, we shall suppose that it is normalized such that $a_{n}$ is positive and we denote this positive leading coefficient by $\kappa_{n}$. With the superstar conjugation, defined by $f^{*}(z)=B_{n}(z) f_{*}(z)$ for $f \in \mathcal{L}_{n} \backslash \mathcal{L}_{n-1}$, we see that $\kappa_{n}=\overline{\phi_{n}^{*}\left(\alpha_{n}\right)}=\phi_{n}^{*}\left(\alpha_{n}\right)>0$.

If we repeat the process for all natural numbers $n$, we obtain an orthonormal system $\left\{\phi_{k}\right\}_{0}^{\infty}$, normalized as above with $\phi_{k} \in \mathcal{L}_{k} \backslash \mathcal{L}_{k-1}, k=1,2, \ldots$ and $\left\langle\phi_{j}, \phi_{k}\right\rangle_{\mu}=\delta_{j k}$.

It is known [5] that the zeros of $\phi_{n}$ are all in $\mathbb{D}$. Thus, they can not be used as nodes in our quadrature. The following two statements are therefore fundamental.

Theorem 2.1 Let $\chi_{n} \in \mathcal{L}_{n} \backslash \mathcal{L}_{n-1}$. Then the following two statements are equivalent

(1) $\chi_{n}$ satisfies

(a) $\chi_{n}^{*}(z)=k \chi_{n}(z), k \neq 0, \forall z \in \mathbb{C} \backslash\left\{1 / \bar{\alpha}_{1}, \ldots, 1 / \bar{\alpha}_{n}\right\}$ (invariance) 
(b) $\left\langle\chi_{n}, f\right\rangle_{\mu}=0, \forall f \in \mathcal{L}_{n-1}\left(\alpha_{n}\right)=\mathcal{L}_{n-1} \cap \mathcal{L}_{n}\left(\alpha_{n}\right)$, where $\mathcal{L}_{k}\left(\alpha_{n}\right)=\left\{g \in \mathcal{L}_{k}: g\left(\alpha_{n}\right)=0\right\}$, and $\left\langle\chi_{n}, 1\right\rangle_{\mu} \cdot\left\langle\chi_{n}, B_{n}\right\rangle_{\mu} \neq 0$ (para-orthogonality)

(2) There exist complex numbers $C_{n} \neq 0$ and $\tau \in \mathbb{T}$ such that $\chi_{n}(z)=C_{n}\left[\phi_{n}(z)+\tau \phi_{n}^{*}(z)\right]$.

Theorem 2.2 Let $\tau \in \mathbb{T}$ and set $\chi_{n}(z)=\chi_{n}(z, \tau)=\phi_{n}(z)+\tau \phi_{n}^{*}(z)$. Then

(1) $\chi_{n}(z)$ has exactly $n$ distinct zeros $z_{1}, \ldots, z_{n}$ which lie on $\mathbb{T}$.

(2) There exist positive numbers $\lambda_{1}, \ldots, \lambda_{n}$ such that

$$
I_{n}(f)=\sum_{j=1}^{n} \lambda_{j} f\left(z_{j}\right)=I_{\mu}(f), \quad \forall f \in \mathcal{R}_{n-1, n-1} .
$$

The $n$-point quadrature formulas (2.3) were introduced by the authors [4] and they represent the extension to the rational case of the so-called Szego" quadrature formulas introduced by Jones et al [12] (see also [9]). For this reason, the formula $I_{n}(f)$ of $(2.3)$ is called a rational Szegő formula and the subspace $\mathcal{R}_{n-1, n-1}$ a maximal domain of validity since there can not exist an $n$-point quadrature rule with nodes on $\mathbb{T}$ and positive weights that is exact in either $\mathcal{R}_{n, n-1}$ or $\mathcal{R}_{n-1, n}$.

Observe that for each $n, I_{n}(f)$ given by (2.3), depends of course on the measure $\mu$, but also on the parameter $\tau$. In this respect we will see how this parameter can be chosen so that the maximal domain of validity meets some extra conditions.

However, before we do that, we first have to say something about the computation of the $I_{n}(f)$. Although we do not elaborate on this, we have to mention that, besides the approach taken below, there are other ways of producing the (rational) Szegö quadrature formulas. For example the approach taken in the polynomial case (i.e., when all $\alpha_{k}=0$ ) via unitary Hessenberg matrices [10] as well as five-diagonal matrices (i.e., using a five-term recurrence relation) as in $[6,2]$, and more recently [7] has been extended to the rational case in the recent paper [15].

From Theorem 2.1, it follows that we need to compute the zeros of $\chi_{n}$, and for that we need, at least in principle, the orthonormal functions $\phi_{n}$. These could be computed from the recurrence relation [5].

Theorem 2.3 For $n \geq 1$, one has

$$
\begin{gathered}
\phi_{n}(z)=\frac{\kappa_{n}}{\kappa_{n-1}}\left[\varepsilon_{n} \frac{\left(z-\alpha_{n-1}\right)}{\left(1-\bar{\alpha}_{n} z\right)} \phi_{n-1}(z)+\delta_{n} \frac{\left(1-\bar{\alpha}_{n-1} z\right)}{\left(1-\bar{\alpha}_{n} z\right)} \phi_{n-1}^{*}(z)\right] \\
\phi_{n}^{*}(z)=-\eta_{n} \frac{\kappa_{n}}{\kappa_{n-1}}\left[\bar{\delta}_{n} \frac{\left(z-\alpha_{n-1}\right)}{\left(1-\bar{\alpha}_{n} z\right)} \phi_{n-1}(z)+\bar{\varepsilon}_{n} \frac{\left(1-\bar{\alpha}_{n-1} z\right)}{\left(1-\bar{\alpha}_{n} z\right)} \phi_{n-1}^{*}(z)\right]
\end{gathered}
$$


with $\alpha_{0}=0$ and $\phi_{0}=\mu_{0}^{-1 / 2}$ with $\mu_{0}=\int_{-\pi}^{\pi} d \mu(\theta)$. Here $\varepsilon_{n} \neq 0$ and $\delta_{n}$ are complex numbers such that $\left|\varepsilon_{n}\right|>\left|\delta_{n}\right|$ and $\eta_{n}=\bar{\alpha}_{n} /\left|\alpha_{n}\right|$ if $\alpha_{n} \neq 0$ and $\eta_{n}=-1$ if $\alpha_{n}=0$.

Thus, once $\phi_{n}$ is computed by (2.4)-(2.5), and given $\tau \in \mathbb{T}$, the nodes of the corresponding rational Szegö formula are computed as the zeros of $\chi_{n}(z, \tau)=\phi_{n}(z)+\tau \phi_{n}^{*}(z)$. However, it will suffice to compute the orthogonal rational functions of degree $n-1$, as shown in the following theorem.

Theorem $2.4 I_{n}(f)=\sum_{j=1}^{n} \lambda_{j} f\left(z_{j}\right)$ is an n-point rational Szegö formula if and only if there exists some $\tau^{*} \in \mathbb{T}$ such that the nodes $\left\{z_{j}\right\}_{j=1}^{n}$ are the zeros of

$$
\left(z-\alpha_{n-1}\right) \phi_{n-1}(z)+\left(1-\bar{\alpha}_{n-1} z\right) \tau^{*} \phi_{n-1}^{*}(z) .
$$

PROOF. Assume $I_{n}(f)=\sum_{j=1}^{n} \lambda_{j} f\left(z_{j}\right)$ is an $n$-point rational Szegő formula. Then there exists a $\tau \in \mathbb{T}$ so that $\left\{z_{j}\right\}_{j=1}^{n}$ are the zeros of $\chi_{n}(z)=\chi_{n}(z, \tau)=\phi_{n}(z)+\tau \phi_{n}^{*}(z)$. Now, by the recurrence (2.4)-(2.5), it follows that

$$
\begin{aligned}
\chi_{n}(z)= & \frac{\kappa_{n}}{\kappa_{n-1}}\left[\varepsilon_{n} \frac{\left(z-\alpha_{n-1}\right)}{\left(1-\bar{\alpha}_{n} z\right)} \phi_{n-1}(z)+\delta_{n} \frac{\left(1-\bar{\alpha}_{n-1} z\right)}{\left(1-\bar{\alpha}_{n} z\right)} \phi_{n-1}^{*}(z)\right] \\
& +\tau \eta_{n} \frac{\kappa_{n}}{\kappa_{n-1}}\left[\bar{\delta}_{n} \frac{\left(z-\alpha_{n-1}\right)}{\left(1-\bar{\alpha}_{n} z\right)} \phi_{n-1}(z)+\bar{\varepsilon}_{n} \frac{\left(1-\bar{\alpha}_{n-1} z\right)}{\left(1-\bar{\alpha}_{n} z\right)} \phi_{n-1}^{*}(z)\right] .
\end{aligned}
$$

Thus

$$
\chi_{n}(z)=\frac{\kappa_{n}}{\kappa_{n-1}\left(1-\bar{\alpha}_{n} z\right)}\left[\left(z-\alpha_{n-1}\right)\left(\varepsilon_{n}+\tau \bar{\delta}_{n} \eta_{n}\right) \phi_{n-1}(z)+\left(1-\bar{\alpha}_{n-1} z\right)\left(\delta_{n}+\tau \bar{\varepsilon}_{n} \eta_{n}\right) \phi_{n-1}^{*}(z)\right] .
$$

Since $\left|\varepsilon_{n}+\tau \bar{\delta}_{n} \eta_{n}\right|>0$ (otherwise $\left.\left|\varepsilon_{n}\right|=\left|\delta_{n}\right|\right)$, we can write

$$
\chi_{n}(z)=\frac{\kappa_{n}\left(\varepsilon_{n}+\tau \bar{\delta}_{n} \eta_{n}\right)}{\kappa_{n-1}\left(1-\bar{\alpha}_{n} z\right)}\left[\left(z-\alpha_{n-1}\right) \phi_{n-1}(z)+\tau^{*}\left(1-\bar{\alpha}_{n-1} \eta_{n}\right) \phi_{n-1}^{*}(z)\right] .
$$

It is obvious that $\left|\tau^{*}\right|=1$. Indeed

$$
\tau^{*}=\tau \eta_{n} \frac{\overline{\tau \eta}_{n} \delta_{n}+\bar{\varepsilon}_{n}}{\varepsilon_{n}+\tau \eta_{n} \bar{\delta}_{n}} \in \mathbb{T}
$$

and the result follows. 
The converse result is proved in a similar way.

\section{Maximal domains}

In an $n$-point rational Szegö quadrature formula, one has $2 n$ parameters: the $n$ nodes and the $n$ weights. Therefore one would expect that it is possible to fix these parameters such that the quadrature formula integrates exactly all functions in a space of dimension $2 n$ as in the case of the classical Gauss formulas, for which it is known that an $n$-point Gauss quadrature formula integrates exactly all polynomials of degree at most $2 n-1$, i.e., all polynomials in $\Pi_{2 n-1}$ with $\operatorname{dim}\left(\Pi_{2 n-1}\right)=2 n$.

On the other hand it is known that an $n$-point rational Szegő formula integrates exactly all functions from $\mathcal{R}_{n-1, n-1}$, a space of dimension $2 n-1$ and this space is a maximal domain of validity in the sense that there is no $n$-point quadrature formula of the form (2.3) that is exact in $\mathcal{R}_{n, n-1}$ or in $\mathcal{R}_{n-1, n}$. A fortiori, the same type of conclusion can be drawn in the polynomial case, that is when all $\alpha_{j}=0$, then the Szego" quadrature formula will integrate exactly all Laurent polynomials in $\operatorname{span}\left\{z^{j}:-(n-1) \leq j \leq n-1\right\}$ and that is a maximal domain of validity.

In these results however it is not said that there does not exist a subspace of dimension $2 n$ where the quadrature formulas are exact, it is only claimed that it can not be of the form $\mathcal{R}_{n, n-1}$ or $\mathcal{R}_{n-1, n}$.

In this section we will characterize a subspace of dimension $2 n$ for which the rational Szegö quadrature formula is exact. A similar result in the polynomial case when all $\alpha_{j}=0$ was obtained in [14].

Suppose that $I_{n}(f)$ is an $n$-point Szegö quadrature formula. Then

$$
I_{n}(f)=I_{\mu}(f), \quad \forall f \in \mathcal{R}_{n-1, n-1}=\operatorname{span}\left\{B_{-(n-1)}, \ldots, B_{n-1}\right\}
$$

where as before $B_{-k}=B_{k *}=1 / B_{k}$. We cannot have in addition that $I_{n}\left(B_{n}\right)=I_{\mu}\left(B_{n}\right)$ or $I_{n}\left(B_{-n}\right)=I_{\mu}\left(B_{-n}\right)$. The key to the construction of a domain of validity with dimension $2 n$ is to use para-orthogonal functions. These have a free parameter $\tau \in \mathbb{T}$ and we shall be able to choose it such that the dimension of the domain of validity can be increased from $2 n-1$ to $2 n$. 
First we note that $\mathcal{R}_{n-1, n-1}$ is also spanned by $\left\{\rho_{j}\right\}_{j=-(n-1)}^{n-1}$ where

$$
\left.\begin{array}{l}
\rho_{j}=\bar{\sigma}_{j} B_{j}-\sigma_{j} B_{-j}, \quad j=0,1, \ldots, n-1 \\
\rho_{-j}=\bar{\sigma}_{j} B_{j}+\sigma_{j} B_{-j}, \quad j=1,2, \ldots, n-1
\end{array}\right\}, \quad \begin{aligned}
& \sigma_{j} \in \mathbb{T}, \quad j=0,1, \ldots, n-1, \\
& \sigma_{0} \neq \pm 1 .
\end{aligned}
$$

Hence, for any $n$-point rational Szegö formula $I_{n}$, it holds that $I_{n}\left(\rho_{j}\right)=I_{\mu}\left(\rho_{j}\right)$, for all $j=$ $0, \pm 1, \ldots, \pm(n-1)$. Now for $n>0$ we will try to find some $\sigma_{n} \in \mathbb{T}$ and an $n$-point Szegö quadrature formula $I_{n}^{-}$such that in addition to the previous properties, it also holds that $I_{n}^{-}\left(\rho_{n}\right)=I_{\mu}\left(\rho_{n}\right)$ for $\rho_{n}=\bar{\sigma}_{n} B_{n}-\sigma_{n} B_{-n}$.

Let $I_{n}^{-}(\cdot)$ be a rational Szegő quadrature formula such that its nodes are given as the zeros of the para-orthogonal function

$$
q_{n}(z)=\bar{s}_{n} \phi_{n}(z)-s_{n} \phi_{n}^{*}(z)=\overline{q_{n}^{*}\left(\alpha_{n}\right)} B_{n}(z)+\cdots+q_{n}\left(\alpha_{1}\right) B_{0}, \quad s_{n} \in \mathbb{T} .
$$

(Observe that, by Theorem 2.2, this means the nodes are produced as the zeros of $\phi_{n}(z)+\tau_{n} \phi_{n}^{*}(z)$ with $\tau_{n}=-s_{n}^{2}$.) Then

$$
B_{n}(z)-\frac{q_{n}(z)}{\overline{q_{n}^{*}\left(\alpha_{n}\right)}} \in \mathcal{L}_{n-1}
$$

Thus this function can be integrated exactly.

$$
I_{\mu}\left(B_{n}-\frac{q_{n}}{\overline{q_{n}^{*}\left(\alpha_{n}\right)}}\right)=I_{n}^{-}\left(B_{n}\right)-\frac{I_{n}^{-}\left(q_{n}\right)}{\overline{q_{n}^{*}\left(\alpha_{n}\right)}}=I_{n}^{-}\left(B_{n}\right)
$$

because the zeros of $q_{n}$ are the nodes of the quadrature formula. Therefore the error $E_{n}\left(B_{n}\right)=$ $I_{\mu}\left(B_{n}\right)-I_{n}^{-}\left(B_{n}\right)$ is given by

$$
E_{n}\left(B_{n}\right)=\frac{I_{\mu}\left(q_{n}\right)}{\overline{q_{n}^{*}\left(\alpha_{n}\right)}}
$$

Now for $n \geq 1$, by orthogonality

$$
I_{\mu}\left(q_{n}\right)=\bar{s}_{n} I_{\mu}\left(\phi_{n}\right)-s_{n} I_{\mu}\left(\phi_{n}^{*}\right)=\bar{s}_{n}\left\langle\phi_{n}, 1\right\rangle_{\mu}-s_{n}\left\langle B_{n}, \phi_{n}\right\rangle_{\mu}=-s_{n} \frac{\left\|\phi_{n}\right\|^{2}}{\kappa_{n}}
$$


where $\phi_{n}=\kappa_{n} B_{n}+\ldots+\phi_{n}\left(\alpha_{1}\right) B_{0}$. Hence

$$
E_{n}\left(B_{n}\right)=-\frac{s_{n}}{\overline{q_{n}^{*}\left(\alpha_{n}\right)}} \frac{\left\|\phi_{n}\right\|^{2}}{\kappa_{n}} .
$$

On the other hand

$$
\frac{1}{B_{n}(z)}\left(1-\frac{q_{n}(z)}{q_{n}\left(\alpha_{n}\right)}\right)=\frac{\left(z-\alpha_{n}\right) p_{n-1}(z)}{\omega_{n}(z)}, \quad p_{n-1} \in \Pi_{n-1}
$$

belongs to $\mathcal{L}_{(n-1) *}$ so that also this function will be integrated exactly. This implies

$$
I_{\mu}\left(\frac{1}{B_{n}(z)}\left(1-\frac{q_{n}(z)}{q_{n}\left(\alpha_{n}\right)}\right)\right)=I_{n}^{-}\left(\frac{1}{B_{n}}\right)-\frac{1}{q_{n}\left(\alpha_{n}\right)} I_{n}^{-}\left(\frac{q_{n}}{B_{n}}\right)=I_{n}^{-}\left(\frac{1}{B_{n}}\right)
$$

so that

$$
E_{n}\left(\frac{1}{B_{n}}\right)=\frac{1}{q_{n}\left(\alpha_{n}\right)} I_{\mu}\left(\frac{q_{n}}{B_{n}}\right)
$$

As before, for $n \geq 1$, using orthogonality we can evaluate

$$
\begin{aligned}
I_{\mu}\left(\frac{q_{n}}{B_{n}}\right) & =\bar{s}_{n} I_{\mu}\left(\frac{\phi_{n}}{B_{n}}\right)-s_{n} I_{\mu}\left(\frac{\phi_{n}^{*}}{B_{n}}\right) \\
& =\bar{s}_{n}\left\langle\phi_{n}, B_{n}\right\rangle_{\mu}-s_{n}\left\langle 1, \phi_{n}\right\rangle_{\mu}=\bar{s}_{n} \frac{\left\|\phi_{n}\right\|^{2}}{\kappa_{n}},
\end{aligned}
$$

which results in

$$
E_{n}\left(\frac{1}{B_{n}}\right)=\frac{\bar{s}_{n}}{q_{n}\left(\alpha_{n}\right)} \frac{\left\|\phi_{n}\right\|^{2}}{\kappa_{n}} .
$$

Now consider the function

$$
\begin{gathered}
f_{n}(z)=\overline{q_{n}^{*}\left(\alpha_{n}\right)} \bar{s}_{n} B_{n}(z)+q_{n}\left(\alpha_{n}\right) s_{n} B_{-n}(z)=\overline{q_{n}^{*}\left(\alpha_{n}\right)} e^{i \theta_{n}} \rho_{n}(z), \\
\rho_{n}(z)=\bar{\sigma}_{n} B_{n}(z)-\sigma_{n} B_{-n}(z), \quad \sigma_{n}=s_{n} e^{i \theta_{n}} \in \mathbb{T}, \quad \theta_{n}=\arg \left(q_{n}\left(\alpha_{n}\right)\right) .
\end{gathered}
$$


That $\rho_{n}$ has indeed this form follows from the fact that we have chosen $q_{n}$ such that $q_{n}^{*}=-q_{n}$.

By construction it is clear that from (3.2) and (3.3) it follows that $E_{n}\left(f_{n}\right)=E_{n}\left(\rho_{n}\right)=0$. The function $\rho_{n}$ from (3.4) is the one that gives the missing dimension. The rational Szegö formula $I_{n}$ is exact in $\mathcal{M}_{n}^{-}=\operatorname{span}\left\{\rho_{-(n-1)}, \ldots, \rho_{n-1}, \rho_{n}\right\}$.

Similarly, starting from the para-orthogonal function $q_{n}(z)=\bar{s}_{n} \phi_{n}(z)+s_{n} \phi_{n}^{*}(z)$, it can also be checked that a rational Szegö quadrature formula $I_{n}^{+}$can be found whose nodes are the zeros of $q_{n}(z)$ and that integrates exactly $\rho_{-n}(z)=\bar{\sigma}_{n} B_{n}(z)+\sigma_{n} B_{-n}(z)$ with $\sigma_{n}$ given by (3.4), and hence will integrate exactly any function from $\mathcal{M}_{n}^{+}=\operatorname{span}\left\{\rho_{-n}, \ldots, \rho_{n-1}\right\}$.

So we may conclude that the following theorem is proved.

Theorem 3.1 Let $I_{n}^{+}$and $I_{n}^{-}$be rational Szegö quadrature formulas whose $n$ nodes $(n>1)$ are the zeros of the para-orthogonal function $q_{n}^{+}$, resp. $q_{n}^{-}$, with $q_{n}^{ \pm}=\bar{s}_{n} \phi_{n} \pm s_{n} \phi_{n}^{*}, s_{n} \in \mathbb{T}$. Then there exists a subspace $\mathcal{M}_{n}^{+}$, resp. $\mathcal{M}_{n}^{-}$, such that $\mathcal{R}_{n-1, n-1} \subset \mathcal{M}_{n}^{ \pm} \subset \mathcal{R}_{n, n}$ both of dimension $2 n$ and such that $I_{n}^{ \pm}(f)=I_{\mu}(f)$ for all $f \in \mathcal{M}_{n}^{ \pm}$.

These subspaces are given by $\mathcal{M}_{n}^{-}=\operatorname{span}\left\{\rho_{-(n-1)}, \ldots, \rho_{n}\right\} \operatorname{resp} . \mathcal{M}_{n}^{+}=\operatorname{span}\left\{\rho_{-n}, \ldots, \rho_{n-1}\right\}$, where $\rho_{ \pm j}(z)=\bar{\sigma}_{j} B_{j}(z) \mp \sigma_{j} B_{-j}, j=-n, \ldots, n$ with $\sigma_{j} \in \mathbb{T}$ arbitrary for $0, \ldots, n-1$ except for $\sigma_{0} \neq \pm 1$ and $\sigma_{n}=s_{n} q_{n}^{ \pm}\left(\alpha_{n}\right) /\left|q_{n}^{ \pm}\left(\alpha_{n}\right)\right|$.

In short: given $\sigma_{j}, j=0, \ldots, n-1$ and $s_{n}$ all on $\mathbb{T}$, we set $\tau= \pm s_{n}^{2}$ and construct the $n$-point Szegö formula as described in Theorem 2.2. This will give the desired quadrature formulas $I_{n}^{ \pm}$ which are exact in $\mathcal{M}_{n}^{ \pm}$.

Remark 3.2 The relation between the basis $\left\{B_{k}\right\}$ and the basis $\left\{\rho_{k}\right\}$ is given by

$$
\left[\begin{array}{c}
\rho_{k} \\
\rho_{-k}
\end{array}\right]=\left[\begin{array}{cc}
\bar{\sigma}_{k} & -\sigma_{k} \\
\bar{\sigma}_{k} & \sigma_{k}
\end{array}\right]\left[\begin{array}{c}
B_{k} \\
B_{-k}
\end{array}\right], \quad k \neq 0 .
$$

The $\sigma_{0}, \ldots, \sigma_{n-1}$ are arbitrary in $\mathbb{T}$ with $\sigma_{0} \neq \pm 1$, while $\sigma_{n}$ is fixed by the quadrature formula, i.e., by the subspace in which we want it to be exact.

Remark 3.3 Note that for $k=1, \ldots, n$, it holds that $\left(\rho_{k}\right)_{*}=-\rho_{k}$, which implies that these functions are purely imaginary on $\mathbb{T}$ while $\left(\rho_{-k}\right)_{*}=\rho_{-k}$ for $k=1, \ldots, n-1$, so that these basis functions are real on $\mathbb{T}$. For $\rho_{0}$, which is a constant, we can choose it either to be real or to be purely imaginary, depending on whether it is taken to be $\bar{\sigma}_{0} B_{0}-\sigma_{0} B_{0}=-2 i \operatorname{Im}\left(\sigma_{0}\right)$ or $\bar{\sigma}_{0} B_{0}+\sigma_{0} B_{0}=2 \operatorname{Re}\left(\sigma_{0}\right)$. 


\section{Preassigned nodes: The Radau case}

In this section we consider the construction of quadrature rules with a certain number of nodes given in advance. We give an extension to the rational framework on $\mathbb{T}$ of the polynomial situation (i.e., all $\alpha_{k}=0$ ) discussed in [11], [3]. When one or two nodes were prescribed on $\mathbb{T}$, the resulting quadrature formulas with maximal domain of validity are the so-called SzegöRadau and Szegö-Lobatto formules. The case of an arbitrary number of nodes prescribed on $\mathbb{T}$ is still an open problem, even in the polynomial situation.

Let us first consider the case of one fixed point $x_{1} \in \mathbb{T}$ prescribed in advance. One has to find distinct points $z_{j} \in \mathbb{T}, j=1, \ldots, n$ and positive weights $A_{1}, \lambda_{1}, \ldots, \lambda_{n}$ such that

$$
I_{n+1}(f)=A_{1} R\left(x_{1}\right)+\sum_{j=1}^{n} \lambda_{j} R\left(z_{j}\right)=I_{\mu}(R), \quad \forall R \in \mathcal{R}_{p(n), p(n)}
$$

with $p=p(n)$ a nonnegative integer that is as large as possible.

Since we are dealing with $n+1$ nodes, we have an obvious bound for $p(n)$, namely $p(n) \leq n$. If the "optimal" value $p(n)=n$ is reached, then $I_{n+1}(f)$ given by $(4.1)$ is called the $(n+1)$-point rational Szegö-Radau formula with a prescribed node at $x_{1}$. In this case, $\mathcal{R}_{n, n}$ will be called the "maximum domain of validity". The existence of such formulas is straightforward. Indeed, by Theorem 2.2, we know that there exist (1) a number $\tau \in \mathbb{T}$, (2) a set of $n+1$ distinct nodes $z_{j}(\tau) \in \mathbb{T}$ and $(3)$ a corresponding set of weights $\lambda_{j}(\tau)>0, j=1, \ldots, n+1$ such that

$$
I_{n+1}^{(\tau)}(f)=\sum_{j=1}^{n+1} \lambda_{j}(\tau) f\left(z_{j}(\tau)\right)=I_{\mu}(f), \quad \forall f \in \mathcal{R}_{n, n}
$$

and these nodes $\left\{z_{j}(\tau)\right\}_{j=1}^{n+1}$ are the zeros of the para-orthogonal function $\chi_{n+1}(z, \tau)=\phi_{n+1}(z)+$ $\tau \phi_{n+1}^{*}(z)$.

It is a simple matter to check that there will always be some $\tau \in \mathbb{T}$ for which $x_{1}$ is a zero of $\phi_{n+1}(z)+\tau \phi_{n+1}^{*}(z)$. This $\tau$ is given by

$$
\tau=-\frac{\phi_{n+1}\left(x_{1}\right)}{\phi_{n+1}^{*}\left(x_{1}\right)} \in \mathbb{T}
$$


which is indeed of modulus 1 since

$$
|\tau|=\left|\frac{\phi_{n+1}\left(x_{1}\right)}{\phi_{n+1}^{*}\left(x_{1}\right)}\right|=1
$$

since $\left|\phi_{n}^{*}(z)\right|=\left|\phi_{n}(z)\right| \neq 0$ for $z \in \mathbb{T}$ by [5, Cor. 3.1.4].

So we can write

$$
\chi_{n+1}(z, \tau)=\phi_{n+1}(z)+\tau \phi_{n+1}^{*}(z)=\frac{\left(z-x_{1}\right) Q_{n}(z)}{\pi_{n+1}(z)}
$$

where $Q_{n} \in \Pi_{n}$ and $Q_{n}\left(z_{j}\right)=0, j=1, \ldots, n$.

We know that $\chi_{n+1}$ is para-orthogonal with respect to the measure $\mu$. Let us next show that $Q_{n}$ also equals the numerator of a rational function that is para-orthogonal with respect to a rational modification of the measure $\mu$.

We consider the poles $\left\{\alpha_{1}, \ldots, \alpha_{n-1}, \alpha_{n+1}\right\}$ and $\tilde{\mathcal{L}}_{n}$, the corresponding subspace of rational functions, i.e.,

$$
\tilde{\mathcal{L}}_{n}=\operatorname{span}\left\{\tilde{B}_{0}, \tilde{B}_{1}, \ldots, \tilde{B}_{n}\right\}
$$

where

$$
\tilde{B}_{k}=B_{k}, \quad k=0,1, \ldots, n-1 \quad \text { and } \tilde{B}_{n}=B_{n-1} \zeta_{n+1}, \quad \zeta_{n+1}(z)=\eta_{n+1} \frac{\alpha_{n+1}-z}{1-\bar{\alpha}_{n+1} z}
$$

Let

$$
R_{n}(z)=\frac{Q_{n}(z)}{\pi_{n-1}(z)\left(1-\bar{\alpha}_{n+1} z\right)} \in \tilde{\mathcal{L}}_{n}
$$

and define the new measure $\tilde{\mu}$ by

$$
d \tilde{\mu}(\theta)=\left|\frac{z-x_{1}}{1-\bar{\alpha}_{n} z}\right|^{2} d \mu(\theta), \quad z=e^{i \theta} .
$$

Then we have, 
Theorem 4.1 The rational function $R_{n}$ given by (4.4) satisfies

(1) $R_{n}$ is invariant (in $\tilde{\mathcal{L}}_{n}$ )

(2) $\left\langle R_{n}, f\right\rangle_{\tilde{\mu}}=0, \forall f \in \tilde{\mathcal{L}}_{n-1} \cap \tilde{\mathcal{L}}_{n}\left(\alpha_{n+1}\right)=\tilde{\mathcal{L}}_{n-1}\left(\alpha_{n+1}\right)=\mathcal{L}_{n-1}\left(\alpha_{n+1}\right)$

(3) $\left\langle R_{n}, 1\right\rangle_{\tilde{\mu}} \cdot\left\langle R_{n}, \tilde{B}_{n}\right\rangle_{\tilde{\mu}} \neq 0$.

\section{PROOF.}

(1) Invariance follows trivially from the fact that $R_{n}$ has all its zeros on $\mathbb{T}$.

(2) In $\tilde{\mathcal{L}}_{n}$ we consider the basis $\left\{U_{0}, U_{1}, \ldots, U_{n}\right\}$ defined by

$$
U_{0}=1, \quad U_{k}(z)=\frac{z-\alpha_{n+1}}{1-\bar{\alpha}_{k} z} B_{k-1}(z), \quad 1 \leq k \leq n-1, \text { and } U_{n}(z)=\frac{z-\alpha_{n+1}}{1-\bar{\alpha}_{n+1} z} B_{n-1}(z) .
$$

Then $\tilde{\mathcal{L}}_{n-1}=\operatorname{span}\left\{U_{0}, \ldots, U_{n-1}\right\}$ and $\tilde{\mathcal{L}}_{n}\left(\alpha_{n+1}\right)=\operatorname{span}\left\{U_{1}, \ldots, U_{n}\right\}$ so that $\tilde{\mathcal{L}}_{n-1}\left(\alpha_{n+1}\right)=$ $\operatorname{span}\left\{U_{1}, \ldots, U_{n-1}\right\}$.

Thus we must prove that $\left\langle R_{n}, U_{k}\right\rangle_{\tilde{\mu}}=0$ for $1 \leq k \leq n-1$. Indeed,

$$
\begin{aligned}
& \left\langle R_{n}, U_{k}\right\rangle_{\tilde{\mu}}=\int_{-\pi}^{\pi} R_{n}(z) \overline{U_{k}(z)} d \tilde{\mu}(\theta) \\
& =\int_{-\pi}^{\pi} \frac{Q_{n}(z)}{\pi_{n-1}(z)\left(1-\bar{\alpha}_{n+1} z\right)} \overline{\left[\frac{\left(z-\alpha_{n+1}\right) B_{k-1}(z)}{1-\bar{\alpha}_{k} z}\right]}\left|\frac{z-x_{1}}{1-\bar{\alpha}_{n} z}\right|^{2} d \mu(\theta) \\
& =\int_{-\pi}^{\pi} \frac{Q_{n}(z)\left(z-x_{1}\right)}{\pi_{n-1}(z)\left(1-\bar{\alpha}_{n} z\right)\left(1-\bar{\alpha}_{n+1} z\right)} \overline{\left[\frac{\left(z-\alpha_{n+1}\right)\left(z-x_{1}\right) B_{k-1}(z)}{\left(1-\bar{\alpha}_{n} z\right)\left(1-\bar{\alpha}_{k} z\right)}\right]} d \mu(\theta) \\
& =\left\langle\chi_{n+1}, f_{k}\right\rangle_{\mu} \text {, with } f_{k}(z)=\frac{\left(z-\alpha_{n+1}\right)\left(z-x_{1}\right) B_{k-1}(z)}{\left(1-\bar{\alpha}_{k} z\right)\left(1-\bar{\alpha}_{n} z\right)} \text {. }
\end{aligned}
$$

We see that $f_{k} \in \mathcal{L}_{n}$ and $f_{k}\left(\alpha_{n+1}\right)=0$, hence $f_{k} \in \mathcal{L}_{n}\left(\alpha_{n+1}\right)$ for $k=1, \ldots, n-1$. Therefore, it follows that

$$
\left\langle R_{n}, U_{k}\right\rangle_{\tilde{\mu}}=\left\langle\chi_{n+1}, f_{k}\right\rangle_{\mu}=0, \quad k=1, \ldots, n-1 .
$$

(3) Assume that $\left\langle R_{n}, 1\right\rangle_{\tilde{\mu}}=0$. Then $R_{n}$ is orthogonal to $\left\{U_{k}: k=0,1, \ldots, n-1\right\}$, (we have set $\left.U_{0}=1\right)$ that represents a basis of $\tilde{\mathcal{L}}_{n-1}=\mathcal{L}_{n-1}$. Therefore $R_{n}(z)=\gamma_{n} \tilde{\phi}_{n}(z),\left(\gamma_{n} \neq 0\right)$ where $\left\{\tilde{\phi}_{k}: k=0,1, \ldots, n\right\}$ is the orthonormal basis of $\tilde{\mathcal{L}}_{n}$ with respect to the measure $\tilde{\mu}$. This clearly leads to a contradiction since the zeros of $\tilde{\phi}_{n}$ lie in $\mathbb{D}$.

On the other hand, 


$$
\begin{aligned}
& \left\langle R_{n}, \tilde{B}_{n}\right\rangle_{\tilde{\mu}}=\int_{-\pi}^{\pi} R_{n}(z) \overline{\tilde{B}_{n}(z)} d \tilde{\mu}(\theta)=\overline{\int_{-\pi}^{\pi} \overline{R_{n}(z)} \tilde{B}(z) d \tilde{\mu}(\theta)} \\
& =\overline{\int_{-\pi}^{\pi} R_{n *}(z) \tilde{B}_{n}(z) d \tilde{\mu}(\theta)}=\overline{\left\langle R_{n}^{*}, 1\right\rangle_{\tilde{\mu}}}=\overline{\tilde{k}} \overline{\left\langle R_{n}, 1\right\rangle_{\tilde{\mu}}} \neq 0
\end{aligned}
$$

since invariance implies $R_{n}^{*}(z)=\tilde{k} R_{n}(z), \tilde{k} \neq 0$.

Remark 4.2 The previous theorem says that $R_{n}$, whose zeros are the remaining nodes $z_{1}, \ldots, z_{n}$ of the rational Szegö-Radau quadrature formula, is a para-orthogonal function in $\tilde{\mathcal{L}}_{n}$ with respect to the measure $\tilde{\mu}$. This means that there must exist some $\tilde{\tau} \in \mathbb{T}$ such that $R_{n}=\tilde{\phi}_{n}+\tilde{\tau} \tilde{\phi}_{n}^{*}$.

The $\tilde{\tau}$ that we need can be found as follows. Suppose that the orthogonal rational functions $\left\{\phi_{k}\right\}_{0}^{\infty}$ are normalized such that $\phi_{n}=P_{n} / \pi_{n}$ with $P_{n}$ a monic polynomial of exact degree $n$. Then clearly $\phi_{n}(0)=P_{n}(0)=\rho_{n}$ with $\rho_{n} \in \mathbb{D}$. On the other hand, recall that

$$
B_{n}(z)=\gamma_{n} \frac{\omega_{n}(z)}{\pi_{n}(z)}, \text { where } \gamma_{n}=(-1)^{n} \prod_{j=1}^{n} \eta_{j}
$$

Thus

$$
\begin{aligned}
\phi_{n}^{*}(z) & =B_{n}(z) \phi_{n *}(z)=B_{n}(z) \frac{\overline{P_{n}(1 / \bar{z})}}{\overline{\pi_{n}(1 / \bar{z})}}=B_{n}(z) \frac{z^{n} P_{n *}(z)}{\omega_{n}(z)}=B_{n}(z) \frac{P_{n}^{*}(z)}{\omega_{n}(z)} \\
& =\gamma_{n} \frac{\omega_{n}(z)}{\pi_{n}(z)} \frac{P_{n}^{*}(z)}{\omega_{n}(z)}=\gamma_{n} \frac{P_{n}^{*}(z)}{\pi_{n}(z)} .
\end{aligned}
$$

Given $\tau \in \mathbb{T}$, we can write without loss of generality that

$$
\chi_{n+1}(z)=\phi_{n+1}(z)+\tau \phi_{n+1}^{*}(z)=\frac{P_{n+1}(z)+\tau \gamma_{n+1} P_{n+1}^{*}(z)}{\pi_{n+1}(z)} .
$$

On the other hand, we also know that

$$
\chi_{n+1}(z)=\frac{\left(z-x_{1}\right)}{\left(1-\bar{\alpha}_{n} z\right)} \frac{Q_{n}(z)}{\pi_{n-1}(z)\left(1-\bar{\alpha}_{n+1} z\right)}=\frac{\left(z-x_{1}\right)}{\left(1-\bar{\alpha}_{n} z\right)} R_{n}(z)
$$


with

$$
R_{n}(z)=\frac{Q_{n}(z)}{\pi_{n-1}(z)\left(1-\bar{\alpha}_{n+1} z\right)}=C_{n}\left[\tilde{\phi}_{n}(z)+\tilde{\tau} \tilde{\phi}_{n}^{*}(z)\right]
$$

Setting

$$
\tilde{\phi}_{n}(z)=\frac{\tilde{P}_{n}(z)}{\pi_{n-1}(z)\left(1-\bar{\alpha}_{n+1} z\right)},
$$

then

$$
R_{n}(z)=C_{n}\left[\frac{\tilde{P}_{n}(z)+\tilde{\tau} \tilde{\gamma}_{n} \tilde{P}_{n}^{*}(z)}{\pi_{n-1}(z)\left(1-\bar{\alpha}_{n+1} z\right)}\right], \quad \tilde{\gamma}_{n}=-\gamma_{n+1} / \eta_{n}
$$

Thus, from (4.7-4.9) it follows that

$$
P_{n+1}(z)+\tau \gamma_{n+1} P_{n+1}^{*}(z)=C_{n}\left(z-x_{1}\right)\left[\tilde{P}_{n}(z)+\tilde{\gamma}_{n} \tilde{\tau} \tilde{P}_{n}^{*}(z)\right] .
$$

Set $\tilde{\rho}_{n}=\tilde{P}_{n}(0)=\tilde{\phi}_{n}(0)$.

Now, by comparing the leading coefficients and the constant terms in (4.10), it follows that

$$
\tilde{\tau}=\frac{\eta_{n}}{\gamma_{n+1}} \cdot \frac{\tilde{\rho}_{n}+c}{1+c \tilde{\tilde{\rho}}_{n}}, \quad c=\frac{1}{x_{1}} \cdot \frac{\gamma_{n+1} \tau+\rho_{n+1}}{1+\gamma_{n+1} \tau \bar{\rho}_{n+1}} .
$$

Finally, observe that $\tau \gamma_{n+1} \in \mathbb{T}$ hence $|c|=1$, and thus also $\tilde{\tau} \in \mathbb{T}$, as was to be expected.

Consider again $\chi_{n+1}=\phi_{n+1}+\tau \phi_{n+1}^{*}$ with $\tau$ given by (4.3), i.e., $x_{1} \in \mathbb{T}$ is a zero of $\chi_{n+1}$. Thus we have $n+1$ distinct nodes on $\mathbb{T}: x_{1}, z_{1}, \ldots, z_{n}$ and $n+1$ positive numbers $A_{1}, \lambda_{1}, \ldots, \lambda_{n}$ such that

$$
I_{n+1}(f)=A_{1} f\left(x_{1}\right)+\sum_{j=1}^{n} \lambda_{j} f\left(z_{j}\right)=I_{\mu}(f), \quad \forall f \in \mathcal{R}_{n, n} .
$$

On the other hand, since $z_{1}, \ldots, z_{n}$ are also the zeros of $R_{n}$, by Theorems 2.2 and 4.1 , there 
exist positive numbers $\tilde{\lambda}_{1}, \ldots, \tilde{\lambda}_{n}$ such that

$$
\tilde{I}_{n}(f)=\sum_{j=1}^{n} \tilde{\lambda}_{j} f\left(z_{j}\right)=I_{\tilde{\mu}}(f), \quad \forall f \in \tilde{\mathcal{R}}_{n-1, n-1} .
$$

Our next step will be to give a simple relation between both sets of weights $\left\{\lambda_{j}\right\}_{1}^{n}$ and $\left\{\tilde{\lambda}_{j}\right\}_{1}^{n}$.

Take into account that the weights can be expressed as

$$
\lambda_{j}=\int_{-\pi}^{\pi} \ell_{j}(z) d \mu(\theta), \quad \ell_{j} \in \mathcal{L}_{n}, \quad \begin{cases}\ell_{j}\left(z_{k}\right)=\delta_{j k}, & 1 \leq j, k \leq n \\ \ell_{j}\left(x_{1}\right)=0, & j=1, \ldots, n\end{cases}
$$

Similarly

$$
\tilde{\lambda}_{j}=\int_{-\pi}^{\pi} \tilde{\ell}_{j}(z) d \tilde{\mu}(\theta), \quad \tilde{\ell}_{j} \in \tilde{\mathcal{L}}_{n-1}, \quad \tilde{\ell}_{j}\left(z_{k}\right)=\delta_{j k}, \quad 1 \leq j, k \leq n .
$$

Now it can be easily checked that

$$
\lambda_{j}=\frac{1}{\left(1-\bar{\alpha}_{n+1} z_{j}\right) \chi_{n+1}^{\prime}\left(z_{j}\right)} \int_{-\pi}^{\pi} \frac{\left(1-\bar{\alpha}_{n+1} z\right) \chi_{n+1}(z)}{z-z_{j}} d \mu(\theta), \quad j=1, \ldots, n
$$

and

$$
\tilde{\lambda}_{j}=\frac{1}{\left(1-\bar{\alpha}_{n+1} z_{j}\right) R_{n}^{\prime}\left(z_{j}\right)} \int_{-\pi}^{\pi} \frac{\left(1-\bar{\alpha}_{n+1} z\right) R_{n}(z)}{z-z_{j}} d \tilde{\mu}(\theta), \quad j=1, \ldots, n .
$$

On the other hand, it also holds that $\chi_{n+1}(z)=\left(z-x_{1}\right) R_{n}(z) /\left(1-\bar{\alpha}_{n} z\right)$, yielding

$$
\chi_{n+1}^{\prime}\left(z_{j}\right)=\left(\frac{z_{j}-x_{1}}{1-\bar{\alpha}_{n} z_{j}}\right) R_{n}^{\prime}\left(z_{j}\right), \quad j=1, \ldots, n .
$$

Hence for $j=1, \ldots, n$, 


$$
\begin{aligned}
\tilde{\lambda}_{j} & =\frac{1}{\left(1-\bar{\alpha}_{n+1} z_{j}\right) R_{n}^{\prime}\left(z_{j}\right)} \int_{-\pi}^{\pi}\left(\frac{1-\bar{\alpha}_{n+1} z}{z-z_{j}}\right) R_{n}(z) \overline{\left(\frac{z-x_{1}}{1-\bar{\alpha}_{n} z}\right) \overline{\left(\frac{z-x_{1}}{1-\bar{\alpha}_{n} z}\right)}} d \mu(\theta) \\
& =\frac{1}{\left(1-\bar{\alpha}_{n+1} z\right) R_{n}^{\prime}\left(z_{j}\right)} \int_{-\pi}^{\pi}\left(\frac{1-\bar{\alpha}_{n+1} z}{z-z_{j}}\right) \chi_{n+1}(z) \overline{\left(\frac{z-x_{1}}{1-\bar{\alpha}_{n} z}\right)} d \mu(\theta) .
\end{aligned}
$$

But for $z \in \mathbb{T}$ :

$$
\overline{\left(\frac{z-x_{1}}{1-\bar{\alpha}_{n} z}\right)}=-\bar{x}_{1}+\frac{1-\bar{x}_{1} \alpha_{n}}{z-\alpha_{n}}
$$

Therefore

$$
\begin{aligned}
\tilde{\lambda}_{j}=\frac{-\bar{x}_{1}}{\left(1-\bar{\alpha}_{n+1} z_{j}\right) R_{n}^{\prime}\left(z_{j}\right)} \int_{-\pi}^{\pi} \frac{\left(1-\bar{\alpha}_{n+1} z\right) \chi_{n+1}(z)}{z-z_{j}} d \mu(\theta) \\
+\frac{1-\bar{x}_{1} \alpha_{n}}{\left(1-\bar{\alpha}_{n+1} z_{j}\right) R_{n}^{\prime}\left(z_{j}\right)} \int_{-\pi}^{\pi} \frac{\left(1-\bar{\alpha}_{n+1} z\right) \chi_{n+1}(z)}{\left(z-z_{j}\right)\left(z-\alpha_{n}\right)} d \mu(\theta) .
\end{aligned}
$$

Now

$$
\frac{1}{\left(z-z_{j}\right)\left(z-\alpha_{n}\right)}=\frac{1}{z_{j}-\alpha_{n}}\left[\frac{1}{z-z_{j}}-\frac{1}{z-\alpha_{n}}\right]
$$

yielding

$$
\begin{aligned}
\int_{-\pi}^{\pi} \frac{1-\bar{\alpha}_{n+1} z}{\left(z-z_{j}\right)\left(z-\alpha_{n}\right)} \chi_{n+1}(z) d \mu(\theta)= & \frac{1}{z_{j}-\alpha_{n}} \int_{-\pi}^{\pi}\left(\frac{1-\bar{\alpha}_{n+1} z}{z-z_{j}}\right) \chi_{n+1}(z) d \mu(\theta) \\
& -\frac{1}{z_{j}-\alpha_{n}} \int_{-\pi}^{\pi} \frac{\left(1-\bar{\alpha}_{n+1} z\right) \chi_{n+1}(z)}{z-\alpha_{n}} d \mu(\theta) .
\end{aligned}
$$

The last integral can be written as $\left\langle\chi_{n+1}, f\right\rangle_{\mu}$ with $f=\left(z-\alpha_{n+1}\right) /\left(1-\bar{\alpha}_{n} z\right)$ so that by the para-orthogonality of $\chi_{n+1}$, we conclude that $\left\langle\chi_{n+1}, f\right\rangle_{\mu}=0$.

Finally, from (4.13):

$$
R_{n}^{\prime}\left(z_{j}\right)=\left(\frac{1-\bar{\alpha}_{n} z_{j}}{z_{j}-x_{1}}\right) \chi_{n+1}^{\prime}\left(z_{j}\right), \quad j=1, \ldots, n
$$


Recall that $z_{j} \neq z_{k}, j \neq k, 1 \leq j, k \leq n$ and from (4.14) we conclude that

$$
\tilde{\lambda}_{j}=\lambda_{j}\left[\frac{1-\bar{x}_{1} z_{j}}{1-\bar{\alpha}_{n} z_{j}}+\frac{1-\bar{x}_{1} \alpha_{n}}{z_{j}-\alpha_{n}} \frac{z_{j}-x_{1}}{1-\bar{\alpha}_{n} z_{j}}\right]=\lambda_{j} \frac{2\left[1-\operatorname{Re}\left(z_{j} \bar{x}_{1}\right)\right]}{\left|z_{j}-\alpha_{n}\right|^{2}}, \quad j=1, \ldots, n
$$

Finally, for the weight $A_{1}$ we get

$$
A_{1}=\frac{1}{\left(1-\bar{\alpha}_{n+1} x_{1}\right) \chi_{n+1}^{\prime}\left(x_{1}\right)} \int_{-\pi}^{\pi} \frac{1-\bar{\alpha}_{n+1} z}{z-x_{1}} \chi_{n+1}(z) d \mu(\theta)
$$

Thus, from $\chi_{n+1}(z)=\left(z-x_{1}\right) R_{n}(z) /\left(1-\bar{\alpha}_{n} z\right)$, it follows

$$
A_{1}=\frac{1-\bar{\alpha}_{n} x_{1}}{\left(1-\bar{\alpha}_{n+1} x_{1}\right) R_{n}\left(x_{1}\right)} \int_{-\pi}^{\pi}\left(\frac{1-\bar{\alpha}_{n+1} z}{1-\bar{\alpha}_{n} z}\right) R_{n}(z) d \mu(\theta)
$$

In short: we have seen that the $(n+1)$-point rational Szegö-Radau formula exists and it is uniquely determined from the fixed node $x_{1}$ and that its computation eventually reduces to the computation of an $n$-point Szegö formula for the new measure $d \tilde{\mu}$ as in (4.5) using the poles $\left\{\alpha_{1}, \ldots, \alpha_{n-1}, \alpha_{n+1}\right\}$. In other words, we have to compute the zeros of $R_{n}(z)=\tilde{\phi}_{n}(z)+\tilde{\tau} \tilde{\phi}_{n}^{*}(z)$ for some $\tilde{\tau} \in \mathbb{T}$. However, from Theorem 2.4, we only need to compute the zeros of

$$
\left(z-\alpha_{n-1}\right) \tilde{\phi}_{n-1}(z)+\left(1-\bar{\alpha}_{n-1} z\right) \tilde{\tau}^{*} \tilde{\phi}_{n-1}^{*}(z)
$$

with $\tilde{\tau} \in \mathbb{T}$. Hence, we only need the rational trigonometric moments $\tilde{\mu}_{0}, \ldots, \tilde{\mu}_{n-1}$ where

$$
\tilde{\mu}_{k}=\int_{-\pi}^{\pi} \overline{\tilde{B}_{k}(z)} d \tilde{\mu}(\theta)=\int_{-\pi}^{\pi} \overline{B_{k}(z)} d \tilde{\mu}(\theta), \quad k=0,1, \ldots, n-1
$$

Clearly, this will only work if we know which $\tilde{\tau}^{*}$ to choose. Indeed, we take $\tau$ given by (4.3) and obtain $\tilde{\tau}$ by (4.11). Finally $\tilde{\tau}^{*}$ is deduced by conveniently using formula (2.8). 


\section{$5 \quad$ The link with reproducing kernels}

In the spaces $\mathcal{L}_{n}$ with orthonormal basis functions $\left\{\phi_{j}\right\}_{j=0}^{n}$, it is well known that $k_{n}(z, w)=$ $\sum_{j=0}^{n} \phi_{j}(z) \overline{\phi_{j}(w)}$ are reproducing kernels in the sense that $\left\langle f(\cdot), k_{n}(\cdot, w)\right\rangle_{\mu}=f(w)$ for all $f \in \mathcal{L}_{n}$. Moreover these kernels satisfy the Christoffel-Darboux relations:

$$
\begin{aligned}
k_{n}(z, w) & =\frac{\phi_{n+1}^{*}(z) \overline{\phi_{n+1}^{*}(w)}-\phi_{n+1}(z) \overline{\phi_{n+1}(w)}}{1-\zeta_{n+1}(z) \overline{\zeta_{n+1}(w)}} \\
& =\frac{\phi_{n}^{*}(z) \overline{\phi_{n}^{*}(w)}-\zeta_{n}(z) \overline{\zeta_{n}(w)} \phi_{n}(z) \overline{\phi_{n}(w)}}{1-\zeta_{n}(z) \overline{\zeta_{n}(w)}} .
\end{aligned}
$$

Because of these relations, it is easy to make a connection with para-orthogonal functions. Indeed, the numerator

$$
\begin{aligned}
q_{n+1}(z) & =q_{n+1}(z, w)=\phi_{n+1}^{*}(z) \overline{\phi_{n+1}^{*}(w)}-\phi_{n+1}(z) \overline{\phi_{n+1}(w)}, \quad w \in \mathbb{T} \\
& =C_{n+1}\left[\phi_{n+1}(z)+\tau_{n+1} \phi_{n+1}^{*}(z)\right], \quad C_{n+1}=-\overline{\phi_{n+1}(w)} \text { and } \tau_{n+1}=-\frac{\phi_{n+1}(w)}{\phi_{n+1}^{*}(w)} \in \mathbb{T}
\end{aligned}
$$

is obviously invariant, and para-orthogonal.

Therefore, see Theorem 2.2, its zeros are distinct and on $\mathbb{T}$ and can thus be used as the nodes of an $(n+1)$-point rational Szegő quadrature formula.

Moreover, since

$$
\tau_{n+1}=\tau_{n+1}(w)=-\frac{\phi_{n+1}(w)}{\phi_{n+1}^{*}(w)} \in \mathbb{T}, \quad \forall w \in \mathbb{T}
$$

and because $\phi_{n+1}(t)+\tau \phi_{n+1}^{*}(t)$ is para-orthogonal, and therefore has $n+1$ zeros which are all in $\mathbb{T}$, it is clear that there are for any $\tau \in \mathbb{T}$ precisely $n+1$ distinct values of $w \in \mathbb{T}$ such that $\tau_{n+1}(w)=\tau$. Because the para-orthogonal functions of a certain degree form a one parameter family, fixing this parameter by choosing one of its zeros will automatically fix the other zeros. In other words, for any $(n+1)$-point rational Szegö quadrature formula there will exist $n+1$ distinct values of $w$ such that the nodes of the quadrature formula are the zeros of a para-orthogonal function of the form $q_{n+1}$. 
Thus the nodes of that rational Szegö-Radau quadrature formula with one prefixed node $x_{1} \in \mathbb{T}$ will be the zeros of $q_{n+1}$ where $w$ should be chosen such that $x_{1}$ is one of these zeros. Clearly $z=w$ is a zero of $q_{n+1}(z)$, so that the nodes of the rational Szegö-Radau quadrature formula with prefixed node $x_{1} \in \mathbb{T}$, will be found as the zeros of $q_{n+1}\left(z, x_{1}\right)$.

By the first Christoffel-Darboux relation, we see, that these nodes are also the zeros of

$$
\left(1-\zeta_{n+1}(z) \overline{\zeta_{n+1}\left(x_{1}\right)}\right) k_{n}\left(z, x_{1}\right)
$$

Thus to get the nodes $z_{1}, \ldots, z_{n}$ of the rational Szegö-Radau quadrature formula that are different from $x_{1}$, we need to find the zeros of $k_{n}\left(z, x_{1}\right)$.

Thus to find the nodes of the rational Szegö-Radau quadrature formula with prefixed node $x_{1} \in \mathbb{T}$, we could compute $\phi_{j}(z)$ and $\phi_{j}\left(x_{1}\right)$ for $j=0,1, \ldots, n$ by the recurrence relation, form $k_{n}\left(z, x_{1}\right)=\sum_{j=0}^{n} \phi_{j}(z) \overline{\phi_{j}\left(x_{1}\right)}$ and compute its zeros.

Also the result of Theorem 2.4 can be recovered using the second Christoffel-Darboux relation. Indeed,

$$
\begin{aligned}
q_{n+1}(z, w) & =\left(1-\zeta_{n+1}(z) \overline{\zeta_{n+1}\left(x_{1}\right)}\right) k_{n}\left(z, x_{1}\right) \\
& =\frac{1-\zeta_{n+1}(z) \overline{\zeta_{n+1}\left(x_{1}\right)}}{1-\zeta_{n}(z) \overline{\zeta_{n}\left(x_{1}\right)}}\left[\phi_{n}^{*}(z) \overline{\phi_{n}^{*}(w)}-\zeta_{n}(z) \overline{\zeta_{n}(w)} \phi_{n}(z) \overline{\phi_{n}(w)}\right] \\
& =\frac{c_{n}(w)}{1-\bar{\alpha}_{n+1} z}\left[\left(z-\alpha_{n}\right) \phi_{n}(z)+\left(1-\bar{\alpha}_{n} z\right) \tilde{\tau}_{n}(w) \phi_{n}^{*}(z)\right]
\end{aligned}
$$

with $($ for $w \in \mathbb{T})$

$$
c_{n}(w)=\frac{\left(1-\left|\alpha_{n+1}\right|^{2}\right)\left(x_{1}-\alpha_{n}\right)\left(1-\bar{\alpha}_{n} w\right)}{\left(1-\left|\alpha_{n}\right|^{2}\right)\left(x_{1}-\alpha_{n+1}\right)\left(\alpha_{n}-w\right)} \overline{\phi_{n}(w)}, \quad \overline{\tilde{\tau}_{n}(w)}=\frac{\left(1-\bar{\alpha}_{n} w\right) \phi_{n}^{*}(w)}{\left(\alpha_{n}-w\right) \phi_{n}(w)} \in \mathbb{T} .
$$

Thus the zeros of $k_{n}(z, w)$ can also be found as the zeros of $\left(z-\alpha_{n}\right) \phi_{n}(z)+\left(1-\bar{\alpha}_{n} z\right) \tilde{\tau} \phi_{n}^{*}(z)$ where $\tilde{\tau}=\tilde{\tau}_{n}(w)$ is as we described above. 


\section{Rational Szegö-Lobatto formulas}

Throughout this section we will assume that $x_{1}$ and $x_{2}$ are given on $\mathbb{T}$ such that $x_{1} \neq x_{2}$. We shall be concerned with the problem of finding $n$ distinct points $z_{1}, \ldots, z_{n}$ also on $\mathbb{T}$ and all different from $x_{1}$ and $x_{2}$ and positive weights $A_{1}, A_{2}, \lambda_{1}, \ldots, \lambda_{n}$ such that

$$
I_{n+2}(f)=A_{1} f\left(x_{1}\right)+A_{2} f\left(x_{2}\right)+\sum_{j=1}^{n} \lambda_{j} f\left(z_{j}\right)=I_{\mu}(f), \quad \forall f \in \mathcal{R}_{p(n), p(n)}
$$

with $p(n)$ a nonnegative integer as large as possible. First, since we are dealing with $n+2$ nodes, it holds that $p(n) \leq n+1$. On the other hand, $\operatorname{since} \operatorname{dim}\left(\mathcal{R}_{p(n), p(n)}\right)=2 p(n)+1$ and the number of unknowns in $(6.1)$ is $2 n+2$, it seems reasonable to impose that $2 p(n)+1 \geq 2 n+2$, i.e. $p(n) \geq n$, yielding $n \leq p(n) \leq n+1$.

Thus, in case $p(n)=n+1$, then $I_{n+2}(f)$ would be exact in $\mathcal{R}_{n+1, n+1}$ so that we would be dealing with $(n+2)$-point rational Szegö quadrature formula and consequently all the above requirements would be satisfied. On the other hand, the other case, $p(n)=n$ would imply $I_{n+2}(f)=I_{\mu}(f), \forall f \in \mathcal{R}_{n, n}$. Now, from (6.1), we have $2 n+2$ parameters available and $\operatorname{dim}\left(\mathcal{R}_{n, n}\right)=2 n+1$, so that we could also try with a domain like either $\mathcal{R}_{n, n+1}$ or $\mathcal{R}_{n+1, n}$, having both dimension $2 n+1$. However, because of the requirement that the weights in (6.1) should be positive, exactness in $\mathcal{R}_{n+1, n}$ or in $\mathcal{R}_{n, n+1}$ would imply exactness in $\mathcal{R}_{n+1, n+1}$. For this reason, we initially try to find a quadrature rule of the form (6.1) with positive weights to be exact in $\mathcal{R}_{n, n}$. In this case, it will be said that $I_{n+2}(f)$, if it exists, represents a $(n+2)$-point rational Szegő-Lobatto formula for the integral $I_{\mu}(f)$ with prescribed nodes $x_{1}$ and $x_{2}$ on $\mathbb{T}$. Here we have followed the terminology recently introduced in [11] in the polynomial case, i.e., all $\alpha_{k}=0, k=1,2, \ldots$. According to the polynomial situation, eventually exactness in $\mathcal{R}_{n+1, n+1}$ could also be reached.

First we have the following characterization theorem.

Theorem 6.1 Let $x_{1}$ and $x_{2}$ be two distinct points fixed on $\mathbb{T}$, and set

$$
I_{n+2}(f)=A_{1} f\left(x_{1}\right)+A_{2} f\left(x_{2}\right)+\sum_{j=1}^{n} \lambda_{j} f\left(z_{j}\right), \quad n \geq 2
$$


where $\left\{z_{j}\right\}_{j=1}^{n}$ are distinct nodes on $\mathbb{T}$ all different from $x_{1}$ and $x_{2}$. Set

$$
R_{n+2}(z)=\frac{\left(z-x_{1}\right)\left(z-x_{2}\right) \prod_{j=1}^{n}\left(z-z_{j}\right)}{\pi_{n+2}(z)}=\frac{Q_{n+2}(z)}{\pi_{n+2}(z)} \in \mathcal{L}_{n+2} .
$$

Then, $I_{n+2}(f)$ is exact in $\mathcal{R}_{n, n}$ if and only if

(1) $I_{n+2}(f)$ is exact in $\mathcal{R}_{p, q}, p$ and $q$ being arbitrary integers such that $0 \leq p, q \leq n$ and $p+q=n+1$.

(2) $\left\langle R_{n+2}, f\right\rangle_{\mu}=0, \forall f \in \mathcal{L}_{n}$ such that $f\left(\alpha_{n+1}\right)=f\left(\alpha_{n+2}\right)=0$.

PROOF. " $\Rightarrow "$

(1) This follows trivially from $\mathcal{R}_{p, q} \subset \mathcal{R}_{n, n}$.

(2) Conditions (2) are equivalent to

$$
\left\langle R_{n+2}(z), \frac{\left(z-\alpha_{n+2}\right)\left(z-\alpha_{n+1}\right) z^{j}}{\pi_{n}(z)}\right\rangle_{\mu}=0, \quad j=0,1, \ldots, n-2 .
$$

Now,

$$
\left\langle R_{n+2}(z), \frac{\left(z-\alpha_{n+2}\right)\left(z-\alpha_{n+1}\right) z^{j}}{\pi_{n}(z)}\right\rangle_{\mu}=I_{\mu}\left[R_{n+2}(z)\left(\frac{\left(z-\alpha_{n+2}\right)\left(z-\alpha_{n+1}\right) z^{j}}{\pi_{n}(z)}\right)_{*}\right] .
$$

But for $j=0,1, \ldots, n-2$ :

$$
\left(\frac{\left(z-\alpha_{n+2}\right)\left(z-\alpha_{n+1}\right) z^{j}}{\pi_{n}(z)}\right)_{*}=\frac{\left(1-\bar{\alpha}_{n+1} z\right)\left(1-\bar{\alpha}_{n+1} z\right) z^{n-j-2}}{\omega_{n}(z)} .
$$

Therefore,

$$
R_{n+2}(z)\left(\frac{\left(z-\alpha_{n+2}\right)\left(z-\alpha_{n+1}\right) z^{j}}{\pi_{n}(z)}\right)_{*}=\frac{Q_{n+2}(z) z^{n-j-2}}{\omega_{n}(z) \pi_{n}(z)}=g_{n, j}(z) \in \mathcal{R}_{n, n}, \quad j=0, \ldots, n-2 .
$$

Hence

$$
\left\langle R_{n+2}(z), \frac{\left(z-\alpha_{n+2}\right)\left(z-\alpha_{n+1}\right) z^{j}}{\pi_{n}(z)}\right\rangle_{\mu}=I_{\mu}\left(g_{n, j}\right)=I_{n+2}\left(g_{n, j}\right)=0
$$


since $g_{n, j}\left(x_{1}\right)=g_{n, j}\left(x_{2}\right)=g_{n, j}\left(z_{k}\right)=0, k=1, \ldots, n$.

" $\Leftarrow$ " Given the nodes $x_{1}$ and $x_{2}$ and $z_{k}, k=1, \ldots, n$ on $\mathbb{T}$, there exists an $(n+2)$-point quadrature formula of the form (6.1), exact in all $\mathcal{R}_{p, q}$ (observe that $\operatorname{dim}\left(\mathcal{R}_{p, q}\right)=n+2$ and that $\mathcal{R}_{p, q}$ is a Chebyshev subspace on $\mathbb{T}$ ). Thus, take $f \in \mathcal{R}_{n, n}$ and consider $L \in \mathcal{R}_{p, q}$ such that $f\left(x_{j}\right)=L\left(x_{j}\right), j=1,2$ and $f\left(z_{k}\right)=L\left(z_{k}\right), k=1, \ldots, n$. Set $D(z)=f(z)-L(z)$ so that $D(z) \in \mathcal{R}_{n, n}$ vanishes at the nodes of $I_{n+2}$. So we have

$$
I_{\mu}(f)=I_{\mu}(D+L)=I_{\mu}(D)+I_{\mu}(L)=I_{\mu}(D)+I_{n+2}(L)=I_{\mu}(D)+I_{n+2}(f) .
$$

Thus, it remains to show that $I_{\mu}(D)=0$. Now we can write

$$
D(z)=\frac{P(z)}{\omega_{n}(z) \pi_{n}(z)}, \quad P(z)=Q_{n+2}(z) q(z) \in \Pi_{2 n}, \quad q \in \Pi_{n-2} .
$$

Hence

$$
\begin{aligned}
D(z) & =\frac{Q_{n+2}(z)\left(1-\bar{\alpha}_{n+2} z\right)\left(1-\bar{\alpha}_{n+1} z\right) q(z)}{\omega_{n}(z) \pi_{n}(z)\left(1-\bar{\alpha}_{n+1} z\right)\left(1-\bar{\alpha}_{n+2} z\right)}=R_{n+2}(z) \frac{q(z)\left(1-\bar{\alpha}_{n+2} z\right)\left(1-\bar{\alpha}_{n+1} z\right)}{\omega_{n}(z)} \\
& =R_{n+2}(z) h_{n *}(z), \quad h_{n}(z)=\frac{q^{*}(z)\left(z-\alpha_{n+2}\right)\left(z-\alpha_{n+1}\right)}{\pi_{n}(z)}, \quad q^{*} \in \Pi_{n-2} .
\end{aligned}
$$

Thus, by (2) it follows that

$$
I_{\mu}(D)=I_{\mu}\left(R_{n+2} h_{n *}\right)=\left\langle R_{n+2}, h_{n}\right\rangle_{\mu}=0 .
$$

This concludes the proof.

Remark 6.2 Setting $Q_{n+2}(z)=\left(z-x_{1}\right)\left(z-x_{2}\right) Q_{n}(z)$ with $Q_{n}(z)=\prod_{j=1}^{n}\left(z-z_{j}\right)$, then it follows from (2) that for $j=0, \ldots, n-2$

$$
\begin{aligned}
& \left\langle R_{n+2}(z), \frac{\left(z-\alpha_{n+2}\right)\left(z-\alpha_{n+1}\right) z^{j}}{\pi_{n}(z)}\right\rangle_{\mu} \\
& \quad=I_{\mu}\left[\frac{\left(z-x_{1}\right)\left(z-x_{2}\right) Q_{n}(z)}{\pi_{n}(z)\left(1-\bar{\alpha}_{n+2} z\right)\left(1-\bar{\alpha}_{n+1} z\right)}\left(\frac{\left(z-\alpha_{n+2}\right)\left(z-\alpha_{n+1}\right) z^{j}}{\pi_{n}(z)}\right)_{*}\right] \\
& \quad=I_{\mu}\left[Q_{n}(z) \frac{\left(z-x_{1}\right)\left(z-x_{2}\right)}{\left|\pi_{n}(z)\right|^{2}} z^{-j-2}\right] .
\end{aligned}
$$


Thus, it follows easily that (2) is equivalent to the fact that $Q_{n}$ is para-orthogonal with respect to the complex varying measure $\left(z=e^{i \theta}\right)$

$$
d \tilde{\mu}(\theta)=\frac{\left(1-x_{1} \bar{z}\right)\left(z-x_{2}\right)}{\left|\pi_{n}(z)\right|^{2}} d \mu(\theta) \quad \text { or } \quad d \tilde{\mu}(\theta)=\frac{\left(1-x_{2} \bar{z}\right)\left(z-x_{1}\right)}{\left|\pi_{n}(z)\right|^{2}} d \mu(\theta) .
$$

In the case when the nodes $x_{1}$ and $x_{2}$ are complex conjugates, i.e., $x_{1}=e^{i \alpha}$ and $x_{2}=e^{-i \alpha}$, $\alpha \in \mathbb{R}$, then

$$
d \tilde{\mu}(\theta)=\frac{2(\cos \theta-\cos \alpha)}{\left|\pi_{n}\left(e^{i \theta}\right)\right|^{2}} d \mu(\theta) .
$$

Therefore, we have para-orthogonality with respect to a real signed measure so that nothing can be assured about the zeros of $Q_{n}$.

From Theorem 6.1, we see that the rational function $R_{n+2} \in \mathcal{L}_{n+2}$ providing the nodes of the desired quadrature formula must fulfil the $n-1$ orthogonality conditions

$$
\left\langle R_{n+2}(z), \frac{\left(z-\alpha_{n+2}\right)\left(z-\alpha_{n+1}\right) z^{j}}{\pi_{n}(z)}\right\rangle_{\mu}=0, \quad j=0, \ldots, n-2
$$

so that one might wonder which extra conditions could be added to get para-orthogonality. In this respect we have

Proposition 6.3 Let $R_{n+2} \in \mathcal{L}_{n+2}$ satisfy (2) along with the additional requirement

$$
\left\langle R_{n+2}(z), \frac{\left(z-\alpha_{n+2}\right) z^{k}}{\pi_{n+1}(z)}\right\rangle_{\mu}=0, \quad\left\{\begin{array}{l}
k=0,1 \text { if } \alpha_{n+1} \neq 0 \\
k=0, n \text { if } \alpha_{n+1}=0 .
\end{array}\right.
$$

Then $R_{n+2}(z)$ is a para-orthogonal function with respect to the measure $\mu$.

PROOF. We must check that $\left\langle R_{n+2}, f\right\rangle_{\mu}=0, \forall f \in \mathcal{L}_{n+1} \cap \mathcal{L}_{n+2}\left(\alpha_{n+2}\right)$. Assume first that $\alpha_{n+1} \neq 0$ and take $f \in \mathcal{L}_{n+1} \cap \mathcal{L}_{n+2}\left(\alpha_{n+2}\right)$, then

$$
f(z)=\frac{\left(z-\alpha_{n+2}\right) P(z)}{\pi_{n+1}(z)}, \quad P \in \Pi_{n} .
$$


Thus we can write

$$
P(z)=\left(z-\alpha_{n+1}\right)\left(1-\bar{\alpha}_{n+1} z\right) \tilde{P}(z)+A z+B, \quad \tilde{P} \in \Pi_{n-2}, \quad A, B \in \mathbb{C} .
$$

Therefore

$$
\begin{aligned}
\left\langle R_{n+2}, f\right\rangle_{\mu}=\left\langle R_{n+2}(z), \frac{\left(z-\alpha_{n+2}\right)\left(z-\alpha_{n+1}\right) \tilde{P}(z)}{\pi_{n}(z)}\right\rangle_{\mu} & \\
& +\bar{A}\left\langle R_{n+2}(z), \frac{\left(z-\alpha_{n+2}\right) z}{\pi_{n+1}(z)}\right\rangle_{\mu}+\bar{B}\left\langle R_{n+2}(z), \frac{\left(z-\alpha_{n+2}\right)}{\pi_{n+1}(z)}\right\rangle_{\mu}=0
\end{aligned}
$$

by $(2-6.4)$.

Suppose now that $\alpha_{n+1}=0$ (hence that $\pi_{n+1}(z)=\pi_{n}(z)$ ), then

$$
P(z)=A z^{n}+z \tilde{P}(z)+B, \quad \tilde{P} \in \Pi_{n-2}, \quad A, B \in \mathbb{C} .
$$

Hence

$$
f(z)=\frac{\left(z-\alpha_{n+2}\right) P(z)}{\pi_{n+1}(z)}=\frac{A\left(z-\alpha_{n+2}\right) z^{n}}{\pi_{n+1}(z)}+\frac{\left(z-\alpha_{n+2}\right)\left(z-\alpha_{n+1}\right) \tilde{P}(z)}{\pi_{n}(z)}+\frac{B\left(z-\alpha_{n+2}\right)}{\pi_{n+1}(z)} .
$$

it then follows again form (2-6.4) that $\left\langle R_{n+2}, f\right\rangle_{\mu}=0$.

Finally, the remaining condition $\left\langle R_{n+2}, 1\right\rangle_{\mu} \cdot\left\langle R_{n+2}, B_{n+2}\right\rangle \neq 0$ is a straightforward consequence from the assumption that the zeros of $R_{n+2}$ lie on $\mathbb{T}$. See the proof of Theorem 4.1.

Example 6.4 In order to illustrate Theorem 6.1, let us consider the normalized Lebesgue measure, i.e., $d \mu(\theta)=d \theta /(2 \pi)$. Thus, setting $R_{n+2}(z)=Q_{n+2}(z) / \pi_{n+2}(z), Q_{n+2} \in \Pi_{n+2}$, then from (2) it follows that

$$
\left\langle\frac{Q_{n+2}}{\pi_{n+2}}, \frac{\left(z-\alpha_{n+2}\right)\left(z-\alpha_{n+1}\right)}{\pi_{n}} z^{j}\right\rangle_{\mu}=0, \quad j=0, \ldots, n-2
$$

Hence,

$$
\left\langle Q_{n+2}, z^{j}\right\rangle_{\mu_{n}}=0, \quad j=2, \ldots, n, \quad \text { with } d \mu_{n}=\frac{d \theta}{2 \pi\left|\pi_{n}\left(e^{i \theta}\right)\right|^{2}}
$$


or equivalently

$$
\int_{\mathbb{T}} \frac{Q_{n+2}(z) z^{n-j-1}}{\pi_{n}(z) \omega_{n}(z)} d z=0, \quad j=2, \ldots, n .
$$

To fix ideas, take $n=2$ so that (6.7) reduces to the condition

$$
\int_{\mathbb{T}} \frac{Q_{4}(z) z^{-1}}{\pi_{2}(z) \omega_{2}(z)} d z=0
$$

yielding

$$
\frac{Q_{4}(0)}{\alpha_{1} \alpha_{2}}+\frac{Q_{4}\left(\alpha_{1}\right) \alpha_{1}^{-1}}{\pi_{2}\left(\alpha_{1}\right) \omega_{2}^{\prime}\left(\alpha_{1}\right)}+\frac{Q_{4}\left(\alpha_{2}\right) \alpha_{2}^{-1}}{\pi_{2}\left(\alpha_{2}\right) \omega_{2}^{\prime}\left(\alpha_{2}\right)}=0
$$

provided that $0 \neq \alpha_{1} \neq \alpha_{2} \neq 0$.

Suppose we fix $x_{1}=1$ and $x_{2}=-1$ and take $\alpha_{1}=1 / 2$ and $\alpha_{2}=-1 / 2$. then, $\pi_{2}(z)=1-z^{2} / 4$ and $\omega_{2}(z)=z^{2}-1 / 4$. Furthermore, since $Q_{4}\left(x_{1}\right)=Q_{4}\left(x_{2}\right)=0$, we have $Q_{4}(z)=\left(z^{2}-1\right) Q_{2}(z)$, $Q_{2}(z)$ being a monic polynomial of degree 2, namely $Q_{2}(z)=z^{2}+b z+c$. Now from (6.8) it follows that $c=1$, yielding $Q_{2}(z)=z^{2}+b z+1$. Because the zeros of $Q_{2}$ should lie on $\mathbb{T}, Q_{2}$ should be invariant, and thus $b \in \mathbb{R}$. Moreover $|b|<2$, otherwise the zeros of $Q_{2}$ would be real. So that we may conclude that

$$
Q_{4}(z)=\left(z^{2}-1\right)\left(z^{2}+b z+1\right), \quad b \in \mathbb{R}, \quad|b|<2 .
$$

Thus the nodes $z_{1,2}$ are given by

$$
z_{1}=\frac{-b+i \sqrt{4-b^{2}}}{2}, \quad z_{2}=\frac{-b-i \sqrt{4-b^{2}}}{2} .
$$

(Observe that $\left|z_{1}\right|=\left|z_{1}(b)\right|=\left|z_{2}\right|=\left|z_{2}(b)\right|=1$.)

So, by fixing the nodes $x_{1,2}= \pm 1$ and taking the remaining ones $z_{1,2}$ as given by (6.10) (notice that $\left.z_{j} \neq x_{k}, j, k=1,2\right)$ then by Theorem 6.1 there exists a 4-point quadrature rule, exact in $\mathcal{R}_{2,2}$ for any $b \in \mathbb{R}$ with $|b|<2$. However, according to our general analysis, nothing can be 
assured about the positivity of the weights. So, let us check what happens in this particular case. Set

$$
I_{4}(f)=A_{1} f\left(x_{1}\right)+A_{2} f\left(x_{2}\right)+\lambda_{1} f\left(z_{1}\right)+\lambda_{2} f\left(z_{2}\right) .
$$

Since $I_{4}$ is exact in $\mathcal{R}_{1,2}$, the weights can be expressed in terms of the integral

$$
I(\alpha)=\frac{\omega_{1}(\alpha) \pi_{2}(\alpha)}{Q_{4}^{\prime}(\alpha)} \frac{1}{2 \pi i} \int_{\mathbb{T}} \frac{Q_{4}(z)}{(z-\alpha) z \omega_{1}(z) \pi_{2}(z)} d z, \quad \alpha \in \mathbb{T} .
$$

Indeed, $A_{j}=I\left(x_{j}\right), j=1,2$ and $\lambda_{k}=I\left(z_{k}\right), k=1,2$. By (6.12) and after some elementary but rather tedious computations one can check that positivity for the weights is assured provided that $|b|<5 / 4$. For instance, when taking $b=0$, then $z_{1}=i$ and $z_{2}=-i$,

$$
A_{1}=A_{2}=\frac{3}{16}, \quad \lambda_{1}=\frac{\sqrt{5}}{8}, \quad \lambda_{2}=\frac{5-\sqrt{5}}{8} .
$$

From Example 6.4, one sees that even in the simple case of the Lebesgue measure, Theorem 6.1 is of very little practical interest. From an algebraic point of view, our problem reduces to studying the existence and characterization of a rational function $R_{n+2} \in \mathcal{L}_{n+2}$ such that

$$
\begin{aligned}
& R_{n+2}\left(x_{1}\right)=R_{n+2}\left(x_{2}\right)=0 \\
& \left\langle R_{n+2}, \frac{\left(z-\alpha_{n+2}\right)\left(z-\alpha_{n+1}\right) z^{j}}{\pi_{n}(z)}\right\rangle_{\mu}=0, \quad j=0, \ldots, n-2 .
\end{aligned}
$$

Thus, setting $R_{n+2}(z)=\sum_{j=0}^{n+2} c_{j} B_{j}(z)$, we see that (6.13) is a homogeneous linear system with $n+1$ equations and $n+3$ unknowns: $c_{0}, \ldots, c_{n+2}$. Hence (6.13) always admits a nontrivial solution. Furthermore, this solution should meet the following additional requirements

(1) $c_{n+2} \neq 0$, i.e., $R_{n+2} \in \mathcal{L}_{n+2} \backslash \mathcal{L}_{n+1}$.

(2) The zeros $z_{1}, \ldots, z_{n}$ of $R_{n+2}$ should lie on $\mathbb{T}$ and should be mutually different and different from $x_{1}$ and $x_{2}$.

(3) The weights in the quadrature formula $I_{n+2}$ of the form given in (6.1) must be positive.

In this respect, since the solutions in (6.13) depend on two free parameters, one could conveniently choose them in order to meet with the above requirements. Actually, this was the procedure followed in Example 6.4 so that it does not seem to be an advisable procedure in 
the general situation. For our purposes, we will proceed in an alternative way. Taking into account that the zeros of the para-orthogonal rational functions with respect to any measure are distinct and on $\mathbb{T}$, our main aim is imposing para-orthogonality on $R_{n+2}$ (from Proposition 6.3 we see that two conditions are lacking) but now with respect to a new measure $\tilde{\mu}$ such that $I_{\mu}(f)=I_{\tilde{\mu}}(f), \forall f \in \mathcal{R}_{n, n}$. Thus, let $\left\{\phi_{k}\right\}_{0}^{\infty}$ be a sequence of orthonormal rational Szego" functions for the measure $d \mu$ and $\left\{\varphi_{k}\right\}_{0}^{\infty}$ is the corresponding sequence of monic functions, i.e., $\varphi_{k}=\phi_{k} / \kappa_{k}, \kappa_{k}=\phi_{k}^{*}\left(\alpha_{k}\right)>0$. Then the recurrence relations (2.4-2.5) become

$$
\begin{gathered}
\varphi_{n}(z)=\varepsilon_{n}\left[\left(\frac{z-\alpha_{n-1}}{1-\bar{\alpha}_{n} z}\right) \varphi_{n-1}(z)+\left(\frac{\delta_{n}}{\varepsilon_{n}}\right)\left(\frac{1-\bar{\alpha}_{n-1} z}{1-\bar{\alpha}_{n} z}\right) \varphi_{n}^{*}(z)\right], \\
\varphi_{n}^{*}(z)=-\eta_{n} \bar{\varepsilon}_{n}\left[\overline{\left(\frac{\delta_{n}}{\varepsilon_{n}}\right)}\left(\frac{z-\alpha_{n-1}}{1-\bar{\alpha}_{n} z}\right) \varphi_{n-1}(z)+\left(\frac{1-\bar{\alpha}_{n-1} z}{1-\bar{\alpha}_{n} z}\right) \varphi_{n}^{*}(z)\right] .
\end{gathered}
$$

Thus we see that the recurrence relations essentially depend on the parameter $\beta_{n}=\delta_{n} / \varepsilon_{n}$ with $\left|\beta_{n}\right|<1$. By conveniently using the Favard type theorem (see Lemma's 8.1.1, 8.1.2, and 8.1.3 along with Theorem 8.1.4 in [5]), we can prove the following.

Theorem 6.5 Let $\mu$ be a positive Borel measure on $\mathbb{T},\left\{\varphi_{n}\right\}_{0}^{\infty}$ being its sequence of monic rational Szegö functions, given a fixed sequence of poles $\left\{\alpha_{n}\right\}_{1}^{\infty} \subset \mathbb{D}$. Then, given $\beta_{n+1} \in \mathbb{D}$, there exists a positive Borel measure $\tilde{\mu}$ on $\mathbb{T}$ such that if $\left\{\tilde{\varphi}_{n}\right\}_{0}^{\infty}$ represents the corresponding monic sequence, it holds that

(1) $\tilde{\varphi}_{k}(z)=\varphi_{k}(z), k=0,1, \ldots, n$

(2) $\tilde{\varphi}_{n+1}(z)=\tilde{\varepsilon}_{n}\left[\frac{z-\alpha_{n}}{1-\bar{\alpha}_{n+1} z} \varphi_{n}(z)+\beta_{n+1} \frac{1-\bar{\alpha}_{n} z}{1-\bar{\alpha}_{n+1} z} \varphi_{n}^{*}(z)\right], \quad \tilde{\varepsilon}_{n+1} \neq 0$.

Without loss of generality, we can assume that $\tilde{\mu}_{0}=\int d \tilde{\mu}(\theta)=\mu_{0}=\int d \mu(\theta)$ so that it immediately follows that

$$
I_{\mu}(R)=I_{\tilde{\mu}}(R), \quad \forall R \in \mathcal{R}_{n, n}
$$

Take $\tau_{n+1} \in \mathbb{T}$ and set

$$
\tilde{\chi}_{n+2}(z)=\left(z-\alpha_{n+1}\right) \tilde{\varphi}_{n+1}(z)+\left(1-\bar{\alpha}_{n+1} z\right) \tau_{n+1} \tilde{\varphi}_{n+1}^{*}(z)
$$


Then from Theorem 2.4 one knows that $\tilde{\chi}_{n+1}$ has $n+2$ distinct zeros $\tilde{z}_{1}, \ldots, \tilde{z}_{n+2}$ on $\mathbb{T}$ and that there exist positive numbers $\tilde{\lambda}_{1}, \ldots, \tilde{\lambda}_{n+2}$ such that

$$
\tilde{I}_{n+2}(f)=\sum_{j=1}^{n+2} \tilde{\lambda}_{j} f\left(\tilde{z}_{j}\right)=I_{\tilde{\mu}}(f), \quad \forall f \in \mathcal{R}_{n+1, n+1} .
$$

Hence, by (6.15) it follows that

$$
\tilde{I}_{n+2}(f)=I_{\mu}(f), \quad \forall f \in \mathcal{R}_{n, n}
$$

In short, given $\beta_{n+1} \in \mathbb{D}$ and $\tau_{n+1} \in \mathbb{T}$, an $(n+2)$-point quadrature rule with distinct nodes and positive weights can be obtained such that it integrates exactly any function from $\mathcal{R}_{n, n}$. Therefore, the problem of constructing a rational Szegö-Lobatto quadrature formula can be reformulated as follows.

Problem 6.6 Given a natural number $n \geq 2$ and two numbers $x_{1}, x_{2} \in \mathbb{T}$ with $x_{1} \neq x_{2}$, along with a positive measure $\mu$, find $\beta \in \mathbb{D}$ and $\tau \in \mathbb{T}$ such that $\tilde{\chi}_{n+2}\left(x_{1}\right)=\tilde{\chi}_{n+2}\left(x_{2}\right)=0$ where $\tilde{\chi}_{n+2}$ is given by (6.16), i.e.,

$$
\tilde{\chi}_{n+2}(z)=\left(z-\alpha_{n+1}\right) \tilde{\varphi}_{n+1}(z)+\left(1-\bar{\alpha}_{n+1} z\right) \tau \tilde{\varphi}_{n+1}^{*}(z)
$$

with $\tilde{\varphi}_{n+1}$ given by (2) and where for our purposes, we can take without loss of generality $\tilde{\varepsilon}_{n+1}=1$, i.e.,

$$
\tilde{\varphi}_{n+1}(z)=\left(\frac{z-\alpha_{n}}{1-\bar{\alpha}_{n+1} z}\right) \varphi_{n}(z)+\beta\left(\frac{1-\bar{\alpha}_{n} z}{1-\bar{\alpha}_{n+1} z}\right) \varphi_{n}^{*}(z)
$$

$\varphi_{n}$ being the $n$-th monic rational Szegö function for the measure $\mu$.

Example 6.7 Let us illustrate the above approach by taking again the normalized Lebesgue measure $d \mu(\theta)=d \theta /(2 \pi), n=2, x_{1}=-x_{2}=1, \alpha_{1}=-\alpha_{2}=1 / 2$ and $\alpha_{3}=0$. In this case the orthogonal functions are known (see e.g., $[5,16]$ )

$$
\varphi_{n}(z)=\left(1-\left|\alpha_{n}\right|^{2}\right) \frac{z B_{n}(z)}{z-\alpha_{n}}, \quad n=0,1, \ldots,
$$

yielding $\varphi_{n}^{*}(z)=\left(1-\left|\alpha_{n}\right|^{2}\right) /\left(1-\bar{\alpha}_{n} z\right)$. Obviously for our purposes, the factor $1-\left|\alpha_{n}\right|^{2}$ can be 
omitted so that one has

$$
\tilde{\varphi}_{n+1}(z)=\frac{z B_{n}(z)+\beta}{1-\bar{\alpha}_{n+1} z}, \quad \text { and } \quad \tilde{\varphi}_{n+1}^{*}(z)=\tilde{\lambda} \frac{1+z \bar{\beta} B_{n}(z)}{1-\bar{\alpha}_{n+1} z}, \quad|\tilde{\lambda}|=1 .
$$

Hence, by (6.16) and for $\tau \in \mathbb{T}$,

$$
\tilde{\chi}_{n+2}(z)=\frac{\left(z-\alpha_{n+1}\right)\left(z B_{n}(z)+\beta\right)+\tilde{\tau}\left(1-\bar{\alpha}_{n+1} z\right)\left(1+z \bar{\beta} B_{n}(z)\right)}{1-\bar{\alpha}_{n+1} z}
$$

with $\tilde{\tau}=\tau \tilde{\lambda} \in \mathbb{T}$. Since $\tau$ is arbitrary, also $\tilde{\tau}$ is arbitrary, so we shall write $\tau$ instead of $\tilde{\tau}$. Thus, when $\alpha_{n+1}=0$,

$$
\tilde{\chi}_{n+2}(z)=z^{2} B_{n}(z)+\beta z+\tau\left(1+z \bar{\beta} B_{n}(z)\right)=\frac{Q_{n+2}(z)}{\pi_{n}(z)}
$$

where, with $\gamma_{2}$ as in (4.6),

$$
Q_{n+2}(z)=z^{2} \gamma_{n} \omega_{n}(z)+\beta z \pi_{n}(z)+\tau\left(\pi_{n}(z)+z \bar{\beta} \gamma_{n} \omega_{n}(z)\right) .
$$

For $n=2$, it results in

$$
Q_{4}(z)=z^{2} \gamma_{2} \omega_{2}(z)+\beta z \pi_{2}(z)+\tau\left(\pi_{2}(z)+z \bar{\beta} \gamma_{2} \omega_{2}(z)\right)
$$

with $\gamma_{2}=-1, \omega_{2}(z)=\left(4 z^{2}-1\right) / 4$, and $\pi_{2}(z)=\left(4-z^{2}\right) / 4$. Hence

$$
4 Q_{4}(z)=z^{2}\left(4 z^{2}-1\right)-\beta z\left(4-z^{2}\right)-\tau\left[4-z^{2}-\bar{\beta} z\left(4 z^{2}-1\right)\right] .
$$

Now, by imposing that $Q_{4}(1)=Q_{4}(-1)=0$, one obtains

$$
\left.\begin{array}{l}
1-\beta-\tau(1-\bar{\beta})=0 \\
1+\beta-\tau(1+\bar{\beta})=0
\end{array}\right\}
$$

yielding

$$
\beta-\bar{\beta}=0 \quad \text { and } \quad \tau=1 .
$$


So $\beta \in \mathbb{R}$, and since we need $|\beta|<1$, we may conclude $\beta \in(-1,1)$.

As a conclusion, by taking $\beta \in(-1,1)$, a four point rational Szegö-Lobatto formula can be obtained. In this case, the polynomial providing the four nodes is

$$
Q_{4}(z)=z^{4}+\frac{5}{4} \beta z^{3}-\frac{5}{4} \beta z-1 .
$$

Since $Q_{4}(1)=Q_{4}(-1)=0$, we get

$$
Q_{4}(z)=\left(z^{2}-1\right)\left(z^{2}+\frac{5}{4} \beta z+1\right)=\left(z^{2}-1\right)\left(z^{2}+b z+1\right)
$$

where $b=5 \beta / 4$. Hence $|b|<5 / 4$ and we come to the same conclusion as in Example 6.4.

The Example 6.7 solves the problem 6.6 for $n=2$ and $\alpha_{1}=-\alpha_{2}=1 / 2$ in the case of the Lebesgue measure. For a general measure, $n=2$ and $\alpha_{1}=\alpha_{2}=0$ (polynomial situation), the problem has been successfully solved by Jagels and Reichel in their recent paper [11]. The analysis and study of Problem 6.6 in its full generality involving an arbitrary measure, any sequence of poles $\left\{\alpha_{k}\right\} \subset \mathbb{D}$ along with an arbitrary number of nodes $z_{1}, \ldots, z_{n}, n \geq 2$ to be determined and two arbitrary prescribed nodes $x_{1}$ and $x_{2}$ on $\mathbb{T}$ is yet unsolved.

On the other hand, a rational Gauss-Lobatto formula for integration over the interval $[-1,1]$ with the Chebyshev weight where the endpoints -1 and 1 were preassigned nodes was considered by G. Min [13].

\section{References}

[1] N.I. Achieser. Theory of approximation. Frederick Ungar Publ. Co., New York, 1956.

[2] G.S. Ammar, W.B. Gragg, and L. Reichel. Constructing a unitary Hessenberg matrix from spectral data. In G. Golub and P. Van Dooren, editors, Numerical linear algebra, digital signal processing and parallel algorithms, volume 70 of NATO-ASI Series, F: Computer and Systems Sciences, pages 385-395, Berlin, 1991. Springer.

[3] A. Bultheel, L. Daruis, and P. González-Vera. Quadrature formulas on the unit circle with prescribed nodes and maximal domain of validity. 2007. In preparation.

[4] A. Bultheel, P. González-Vera, E. Hendriksen, and O. Njåstad. Orthogonal rational functions and quadrature on the unit circle. Numer. Algorithms, 3:105-116, 1992. 
[5] A. Bultheel, P. González-Vera, E. Hendriksen, and O. Njåstad. Orthogonal rational functions, volume 5 of Cambridge Monographs on Applied and Computational Mathematics. Cambridge University Press, 1999.

[6] A. Bunse-Gestner and L. Elsner. Schur parameters pencils for the solution of the unitary eigenproblem. Linear Algebra Appl., 154-156:741-778, 1991.

[7] M.J. Cantero, L. Moral, and L. Velázquez. Measures and para-orthogonal polynomials on the unit circle. East J. Approx., 8:447-464, 2002.

[8] W.B. Gragg. Positive definite Toeplitz matrices, the Arnoldi process for isometric operators and Gaussian quadrature on the unit circle. In E.S. Nikolaev, editor, Numerical methods in linear algebra, pages 16-32. Moscow university press, 1982. (In Russian).

[9] W.B. Gragg. Positive definite Toeplitz matrices, the Arnoldi process for isometric operators, and Gaussian quadrature on the unit circle. J. Comput. Appl. Math., 46, 1993.

[10] W.B. Gragg and L. Reichel. A divide and conquer method for unitary orthogonal eigenproblems. Numer. Math., 57:695-718, 1990.

[11] C. Jagels and L. Reichel. Szegő-Lobatto quadrature rules. J. Comput. Appl. Math., 200(1):116126, 2007.

[12] W.B. Jones, O. Njåstad, and W.J. Thron. Moment theory, orthogonal polynomials, quadrature and continued fractions associated with the unit circle. Bull. London Math. Soc., 21:113-152, 1989.

[13] G. Min. Lobatto type quadrature formulas in rational spaces. J. Comput. Appl. Math., 94:1-12, 1998.

[14] J. Santos-León and O. Njåstad. Domain of validity of Szegő quadrature formulas. J. Comput. Appl. Math., 202(2):440-449, 2007.

[15] L. Velázquez. Spectral methods for orthogonal rational functions. 2007. arXiv:0704.3456v1.

[16] J.L. Walsh. Interpolation and functions analytic interior to the unit circle. Trans. Amer. Math. Soc., 34:523-556, 1932. 